ARTICLE

https://doi.org/10.1038/s41467-019-09283-x

\title{
Microbial recognition by GEF-H1 controls IKKe mediated activation of IRF5
}

Yun Zhao ${ }^{1}$, Rachid Zagani ${ }^{1}$, Sung-Moo Park ${ }^{1}{ }^{1}$, Naohiro Yoshida ${ }^{1}$, Pankaj Shah ${ }^{1} \&$ Hans-Christian Reinecker ${ }^{1}$

During infection, transcription factor interferon regulatory factor 5 (IRF5) is essential for the control of host defense. Here we show that the microtubule-associated guanine nucleotide exchange factor (GEF)-H1, is required for the phosphorylation of IRF5 by microbial muramyldipeptides (MDP), the minimal structural motif of peptidoglycan of both Gram-positive and Gram-negative bacteria. Specifically, GEF-H1 functions in a microtubule based recognition system for microbial peptidoglycans that mediates the activation of IKKE which we identify as a new upstream IKK $\alpha / \beta$ and IRF5 kinase. The deletion of GEF-H1 or dominantnegative variants of GEF-H1 prevent activation of IKKE and phosphorylation of IRF5. The GEF-H1-IKKE-IRF5 signaling axis functions independent of NOD-like receptors and is critically required for the recognition of intracellular peptidoglycans and host defenses against Listeria monocytogenes.

\footnotetext{
${ }^{1}$ Department of Medicine, Gastrointestinal Unit and Center for the Study of Inflammatory Bowel Disease, Massachusetts General Hospital, Harvard Medica School, Boston, MA 02114, USA. These authors contributed equally: Yun Zhao, Rachid Zagani. Correspondence and requests for materials should be addressed to H.-C.R. (email: hans-christian_reinecker@hms.harvard.edu)
} 
$\mathrm{M}$ icrotubules are components of the cytoskeleton that have important functions in cell-autonomous and innate immunity to enable antimicrobial host defense in addition to their roles in control of cell division shape and movement ${ }^{1}$. The guanine nucleotide exchange factor- $\mathrm{H} 1$ (GEF-H1), encoded by ARHGEF2, is a microtubule associated protein that is crucial for sensing intracellular pathogens and initiating transcriptional programs that counter bacterial and viral infections $\mathrm{s}^{2,3}$.

IRF5 is a critical transcription factor regulating immune and inflammatory responses in host defense and disease. Further, polymorphisms in the IRF5 gene have been linked to human autoimmune diseases ${ }^{4-6}$. In addition, IRF5 plays important roles in inflammatory M1-like macrophage polarization ${ }^{7}$. IRF5 has an essential role in the control of inflammation initiated by the activation of RIPK2 in NOD-like receptor (NLR) and IKK $\beta$ during Toll-like (TLR) signaling ${ }^{8,9}$. IKK $\beta$ together with IKKa also mediates NF- $\kappa B$ activation by phosphorylating I $\mathrm{KB}$ proteins as part of the IKK complex together with the regulatory subunit termed IKK $\gamma^{10-13}$.

IKK-related kinases, IKK $\varepsilon^{14,15}$ and TBK1 ${ }^{16-18}$, have been linked to the activation of IRF3 and IRF7 which function as important mediators of antiviral host responses. We have previously shown that GEF-H1 can interact with TBK1 for the activation IRF3 during MAVS-dependent RLR receptor signaling $^{3}$. However, host defense circuits that specifically activate either IKKe or TBK1 have not been specified.

This work uncovers a microtubule based recognition system for microbial peptidoglycan where GEF-H1 is essential for activating the IKK-related kinases, IKKe which we identify as a previously unrecognized IKKa/ $\beta$ and IRF5 kinase. Upon release from microtubules the GEF-H1 signalosome mediates the activation of Rho-Associated, Coiled-Coil Containing Protein Kinases (ROCK) and forms protein complexes that assembled IRF5, $\mathrm{IKK} \varepsilon$, and IKKa/ $\beta$. Although the ability of GEF-H1 to specify IKKe phosphorylation is specific to MDP recognition, IKKe also mediates IRF5 phosphorylation during Toll-like receptor signaling. GEF-H1 mediated IRF5 activation by MDP occurs independent of NOD2. The newly proposed GEF-H1-IKKe-IRF5 signaling axis controls transcriptional programs activated by MDP that define peptidoglycan receptor expression and genes that control intracellular host defense against $L$. monocytogenes in macrophages.

\section{Results}

GEF-H1 is essential for phosphorylation of IRF5 by MDP. To determine the role of GEF-H1 in signaling events induced by MDP, we isolated bone marrow-derived macrophages (BMDMs) from wild-type (WT) C57BL/6, Arhgef2 $2^{-1-}$, and $\mathrm{Nod}^{-1-}$ mice, and determined the phosphorylation and nuclear translocation of IRF5 after stimulation with MDP. As demonstrated in Fig. 1a, lack of GEF-H1 expression impaired IRF5 phosphorylation during MDP-induced immune activation of WT macrophages (Fig. 1a). Surprisingly, IRF5 phosphorylation in response to MDP stimulation occurred unimpeded in $\mathrm{Nod}_{2}^{-1-}$ mice (Fig. 1a). MDP induced Nod2 mRNA expression in WT and Arhgef2 ${ }^{-1-}$ but not in $\mathrm{Nod}^{-I-}$ deficient macrophages within $4 \mathrm{~h}$ (Fig. 1b). Arhgef $2^{-1-}$ macrophages also demonstrated less nuclear translocation of IRF5 compared to WT and Nod2 ${ }^{-1-}$ macrophages upon MDP stimulation (Fig. 1c). GEF-H1 and IRF5 may be able to directly interact as anti-IRF5 but not control IgG pulled down immunocomplexes that contained GEF-H1 from WT but not Arhgef2 ${ }^{-1-}$ macrophages in the absence or presence of MDP (Fig. 1d). IRF5 also became part of protein complexes that were pulled down with anti-GEF-H1 in the presence or absence of
RIPK2 when expressed in HEK293 cells (Fig. 1e). GEF-H1 can interact with RIPK2 which has been identified as a kinase upstream of IKK $\beta$ for the activation of NF- $\mathrm{KB}$ and IRF5 ${ }^{19}$ and we indeed found that Ripk2 ${ }^{-1-}$ macrophages failed to induce IRF5 phosphorylation in response to MDP (Fig. 1f). Furthermore, RIPK2 function was required in MDP stimulated macrophages for the phosphorylation of IKKe and IKKa/ $\beta$ at $\$ 176 / 180$ or S172, respectively (Fig. 1g). We further found that GEF-H1 was able to directly interact with IKKe and IKK $\beta$ in the absence or presence of NOD2 in HEK293T cells (Fig. 1h). Together these data demonstrated that GEF-H1 interacted with RIPK2, IKKe, and IKK $\beta$ for the recruitment and phosphorylation of IRF5 during MDP recognition.

IKKe is required for the phosphorylation of IRF5 by IKK $\alpha / \beta$. Since GEF-H1 interacted with both IKKe and IKK $\beta$, we sought to determine whether IKKe had a role in the phosphorylation of IRF5 during MDP induced cell signaling. We observed enhanced phosphorylation of IRF5 when IKKe but not a kinase deficient IKKe (K38A) variant was co-expressed with GEF-H1 (Fig. 2a). This indicated that GEF-H1 enabled IKKe to function as an upstream IFR5 kinase. As demonstrated in Fig. 2b, IKKe was indeed an essential upstream kinase for the phosphorylation of IRF5 but not NF-kB p65 in response to MDP in macrophages. $I k k^{-1-}$ macrophages were unable to respond to MDP stimulation with the phosphorylation of S445 of IRF5 while phosphorylation of p65 was detectable (Fig. 2b). Further, $I k k^{-1-}$ macrophages failed to activate IKK $\alpha / \beta$ by phosphorylating S176/ 180 in response to MDP (Fig. 2c). In the MDP induced signaling pathway, however, neither TBK1 nor IRF3 were significantly phosphorylated when compared to cyclic di GMP-induced STING signaling (Supplementary Fig. 1). Together these results indicated that IKKe could be a target of RIPK2 for IKKa/ $\beta$ phosphorylation during GEF-H1-dependent IRF5 phosphorylation. Indeed, GEF-H1 and RIPK2 together significantly enhanced IKKe phosphorylation compared to RIPK2 and GEF-H1 expression alone when co-expressed in HEK-293T cells (Fig. 2d). We next determined whether IKKe functioned downstream of RIPK2 in the phosphorylation of IRF5 by overexpressing RIPK2 in the $I k k \varepsilon^{-l-}$ (Fig. 2e) and IKKe in Ripk2 $2^{-l-}$ macrophages (Fig. 2f). RIPK2 induced significant less phosphorylation of IRF5 in

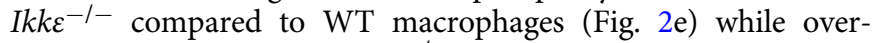
expression of IKKe in Ripk2 ${ }^{-l-}$ macrophages elicited levels of IRF5 phosphorylation that were comparable to those induced in WT macrophages (Fig. 2f). As GEF-H1 is not involved in mediating TLR4 signal transduction ${ }^{3}$, IRF5 phosphorylation occurred comparable in WT and Arhgef2 ${ }^{-1-}$ macrophages in response to LPS (Fig. 2g). In response to LPS, we also observed the acti-

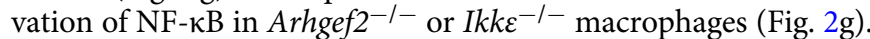
However, IKKe was also required for IRF5 phosphorylation at S445 in response to LPS stimulation of macrophages demonstrating a convergence of distinct pattern recognition pathways activating IKKe for IRF5 phosphorylation (Fig. 2g). Together, these experiments indicated that GEF-H1 specified IKKe function for IKKa/ $\beta$-mediated IRF5 phosphorylation by MDP.

GEF-H1 dephosphorylation is required for IKKe and IRF5 binding. In the next set of experiments, we aimed to define the dephosphorylation events and amino acids of GEF-H1 that are required for the interaction with IKKe and IRF5. Sequence analysis of full-length human GEF-H1 (positional information refers to NP_001155855.1) revealed a pLxIS ( $\mathrm{p}$, hydrophilic residues; $\mathrm{x}$, any amino acid) consensus motif in the C-terminal regions of GEF-H1 at amino acids 320-324 (Fig. 3a). This motif has been proposed to mediate interaction among IRF3, MAVS, and 
a

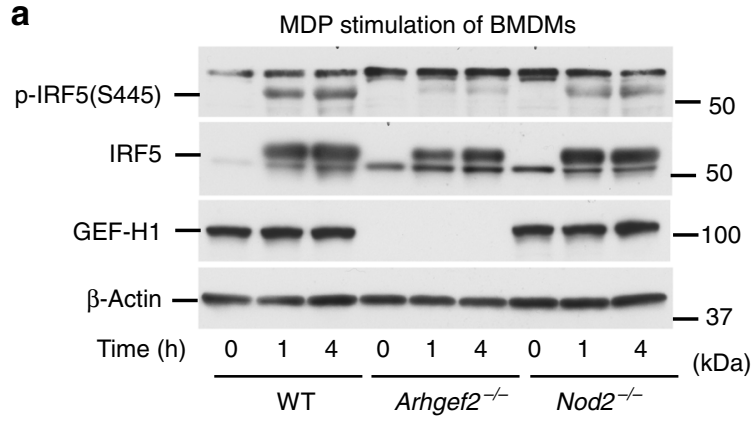

C

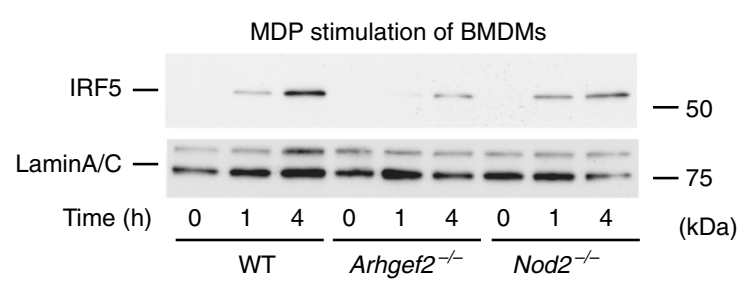

d

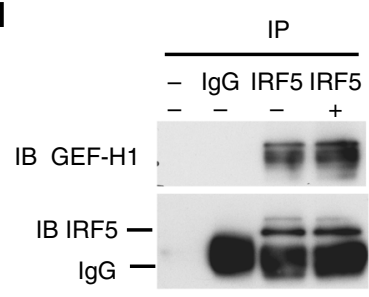

WCL

IB GEF-H1 RT:

IB IRF5

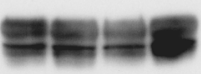

WT BMDMs

f

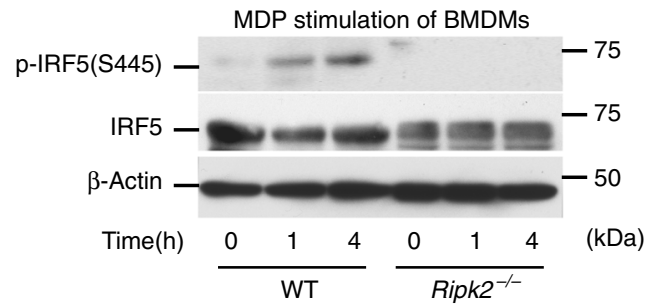

h

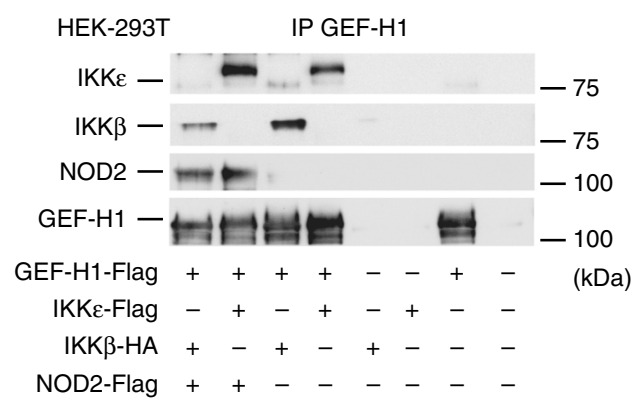

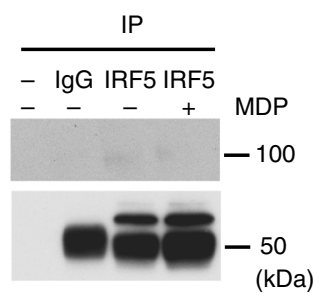

WCL

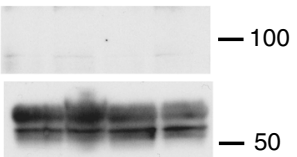

Arhgef $^{-/}$BMDMs $\quad(\mathrm{kDa})$ b

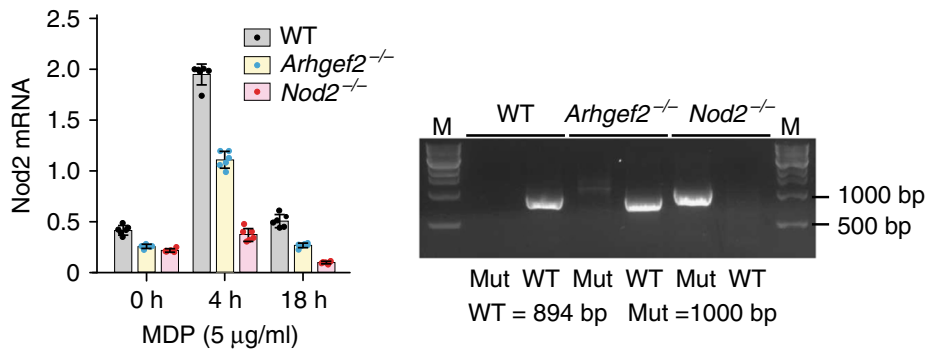

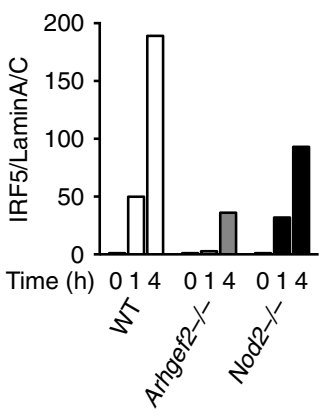

e

HEK-293T IP GEF-H1

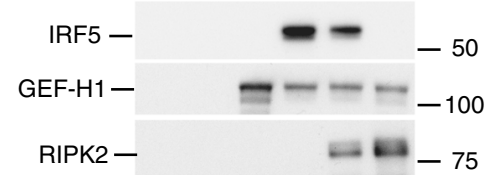

IRF5-GFP $-+-++-\quad(\mathrm{kDa})$

GEF-H1-Flag --++++

RIPK2-HA - $-\ldots+$

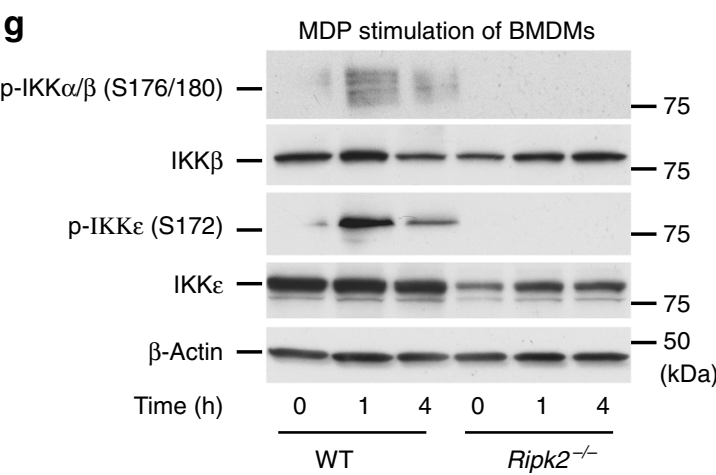

STING $^{20}$. The pLxIS motif in IRF5 has been shown to contain a Serine that is phosphorylated by IKK $\beta$ in response to LPS and is detected by our antibody ${ }^{9,21}$. GEF-H1 contains a second related motif YPLxIS at amino acids 394-399 in which the charged residue in the pLxIS motif is replaced by a Proline but preceded by the Tyrosine at position 394 that is required for exchange function and IRF3 phosphorylation by GEF-H1 in the RIG-I-like receptor signaling 3 . The addition of a phosphate molecule to a non-polar $\mathrm{R}$ group of an amino acid residue could turn ahydrophobic portion of a protein into a polar and extremely hydrophilic one. Figure 3a summarizes the variants created by exchanging the Serines or Tyrosines, respectively, at amino 
Fig. 1 GEF-H1 mediates IRF5 phosphorylation during MDP recognition. a Immunoblot analysis of the activation of IRF5 with antibodies detecting phosphorylated ( $\mathrm{p}-$ ) or total IRF5 in whole-cell lysate of bone marrow derived macrophages (BMDMs) from WT, Arhgef2-/- and Nod2 ${ }^{-/-}$mice after stimulation with $\mathrm{N}$-glycolyl-MDP $\left(5 \mu \mathrm{g} \mathrm{ml}^{-1}\right)$ for $1 \mathrm{~h}$ and $4 \mathrm{~h}$. $\beta$-actin used as loading control. b Nod2 mRNA expression analysis by qRT-PCR from WT, Arhgef2 ${ }^{-/}$and Nod2 ${ }^{-/}$- derived BMDMs and genotyping PCR for targeted or wildtype Nod2 allele in genomic DNA from Nod2 ${ }^{-/-}$, WT and Arhgef2-/mice. c Immunoblot analysis of IRF5 expression in nuclear extracts from BMDMs from WT, Arhgef2 ${ }^{-/-}$and Nod2 ${ }^{-/-}$mice after stimulation with $\mathrm{N}^{-}$ glycolyl-MDP with band intensities quantified using densitometry and Image J software. d Immunoblot analysis of GEF-H1 expression after IRF5 pull down in BMDMs from WT and Arhgef2-/- mice in presence or absence of N-glycolyl-MDP. e Immunoprecipitation of GEF-H1 from HEK293T cells that were transfected with IRF5, GEF-H1 and/or RIPK2 encoding plasmids. $\mathbf{f}$ Western blot analysis of the expression and phosphorylation of IRF5 in BMDMs from WT and Ripk2-/- mice that were stimulated with $\mathrm{N}$-glycolyl-MDP for $1 \mathrm{~h}$ and $4 \mathrm{~h}$. $\mathbf{g}$ Immunoblot analysis of the expression and phosphorylation of IKK $\alpha / \beta$ and IKKE in BMDMs from WT and Ripk2-/- mice after stimulation with $\mathrm{N}$-glycolyl-MDP for $1 \mathrm{~h}$ and $4 \mathrm{~h}$. $\mathbf{h}$ Immunoprecipitation of GEF-H1 with specific

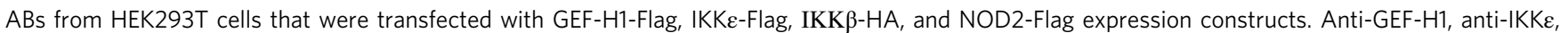
and anti-IKK $\beta$ were used to detect proteins by western blotting and NOD2 was detected with anti-Flag. Source data are provided as a Source Data file

acid 324, 394, 399, and S886 of GEF-H1 to define their relevance for binding and phosphorylating IKKe, IKK $\beta$, and IRF5.

Initially, we determined the phosphorylation status of GEF-H1 variants in HEK293T cells in the absence or presence of IKKe and IKK $\beta$. Surprisingly, modifying Serine 399 of GEF-H1 resulted in a significantly elevated phosphorylation of GEF-H1 at S886 as detected by a specific antibody for this phosphorylated residue (Fig. 3b). Also modifying S324 elevated baseline S886 phosphorylation of GEF-H1, although significantly less compared to S399 (Fig. 3b). The phosphorylation of GEF-H1 at S886 may have important functional consequences, as de-phosphorylation of this residue is required for the release of GEF-H1 from microtubules ${ }^{22}$. IKKe but not IKK $\beta$ further induced additional phosphorylation events that occurred on GEF-H1 and GEF-H1 (Y394A) as indicated by a pan phosphor-serine/threonine antibodies (Fig. 3c).

Remarkably, the hyper-phosphorylated GEF-H1 (S399A) variant was unable to bind IKKe while the exchange deficient GEFH1 mutant Y394A was able to bind IKKe efficiently (Fig. 3d). IKKe binding was also reduced to the GEF-H1 (S324A) variant further indicating that activation of GEF-H1 by dephosphorylation was required to facilitate efficient IKKe binding (Fig. $3 \mathrm{~d}$ ). In contrast, none of these GEF-H1 variants prevented IKK $\beta$ binding to GEF-H1 (Supplementary Fig. 2). To prevent conformational changes in GEF-H1 upon S886 phosphorylation that could interfere with IKKe binding, we created GEF-H1 variants with double substitution of Alanine for S886 and S324 or S399. Coimmunoprecipitations demonstrated that removing the phosphorylation event at $\mathrm{S} 886$ resulted in the increased binding of IKKe to GEF-H1 (Fig. 3e). While the GEF-H1 variant S399A was unable to bind IKKe, the S399A/S886A double mutant interacted with IKKe indicating that binding to GEF-H1 was required for the activation of IKKe by phosphorylation at S172 (Fig. 3e). However, IKKe binding to the GEF-H1 S324A variant also increased when the phosphorylation event at S886 was removed from GEF-H1. GEF-H1 variants that were able to bind IKKe indeed induced the phosphorylation of S172 of IKKe. (Fig. 3e). These experiments also revealed that S324 was particularly important in controlling IKKe phosphorylation as its removal resulted in enhanced phosphorylation of IKKe bound to GEF-H1 (Fig. 3e).

We next determined whether modifying S324 or S399 alone, or in combination with S886 controlled IRF5 binding of GEF-H1. Preventing phosphorylation at S886 resulted in enhanced IRF5 binding to GEF-H1. IRF5 binding was further enhanced to a GEF-H1 variant with Alanine substitutions for S886 and S324. In contrast, exchanging Alanine for S399 in the S886A variant failed to enhance IRF5 binding to GEF-H1 (Fig. 3f). Together, these data demonstrated that the innate immune function of GEF-H1 is controlled by dephosphorylation events that allow the interaction with and phosphorylation of IKKe. Both, S324 and S886 of GEF$\mathrm{H} 1$ are critical in the control of the interaction with IRF5. Further,
S399 within the YPLxIS domain of GEF-H1 plays an important role for the control of phosphorylation events at S886 that inactivate GEF-H1 immune function.

The GEF-H1-IKKe-IRF5 pathway is controlled by ROCK1/2. We co-expressed RFP tagged GEF-H1 and GFP tagged IRF5 together with either IKKe or the kinase deficient IKKe K38A variant to determine subcellular localization of GEF-H1 and nuclear translocation of IRF5 during cell autonomous immune activation by confocal microscopy. GEF-H1 localized to the microtubule network and bundles in the presence of the IKKe K38A variant with IRF5 that was distributed throughout the cytoplasm and enriched in large subcellular compartments (Fig. 4a). When we activated the pathway by expressing functional IKKe, GEF-H1 lost association with the microtubule network and co-localized with IRF5 in small subcellular compartments throughout the cytoplasm (Fig. 4a). Upon coexpression of GEF-H1 together with IKKe, IRF5 translocated to the nucleus (Fig. 4a). Cells that demonstrated nuclear translocation of IRF5 also exhibited membrane blebbing indicative of Rho GTPase function that is enhanced by GEF-H1 and leads to Rho-associated protein kinase (ROCK1) activation ${ }^{2,23}$.

We therefore determined whether GEF-H1 was able to control the phosphorylation of ROCK1 in the context of IKKe. In these experiments we immunoprecipitated ROCK1 from HEK293 cells that were transfected with ROCK1, IKKe, and wild type, and variants of GEF-H1 to determine ROCK1 phosphorylation at S455/T456. Remarkably, the GEF-H1 double-mutant S324A/ S886A allowed the highest phosphorylation of ROCK1 while the S399A/S886A variant failed to induce significant ROCK1 phosphorylation (Fig. 4b). The GEF-H1 variants S324A, S399A, and S886A were able to confer some ROCK1 phosphorylation in the presence of IKKe when compared to wild-type GEF-H1 or the exchange deficient mutant GEF-H1Y394H (Fig. 4b). These experiments indicated that dephosphorylation of GEF-H1 may allow the activation of ROCK1 as a requirement for IRF5 phosphorylation. Indeed, ROCK1 mediated phosphorylation of IKK $\varepsilon$ and IRF5 in the presence of functional IKKe but not inactive IKKe K38A (Fig. 4c). ROCK1 likely functioned downstream of GEF-H1 as during ROCK1 overexpression, GEF-H1 remained associated with microtubules while nuclear IRF5 localization occurred (Fig. 4d). Finally, the ROCK1/2 inhibitor Y27632 inhibited MDP-induced immune activation as IRF5 as well as NF- $\kappa \mathrm{B}$ phosphorylation was reduced in the presence of the inhibitor in WT macrophages (Fig. 4e). Collectively, these data indicated that IKKe and IRF5 activation by GEF-H1 was controlled through ROCK1/2.

GEF-H1 and IRF5 control MDP-initiated transcription. To identify the MDP-induced transcriptional responses that 
a

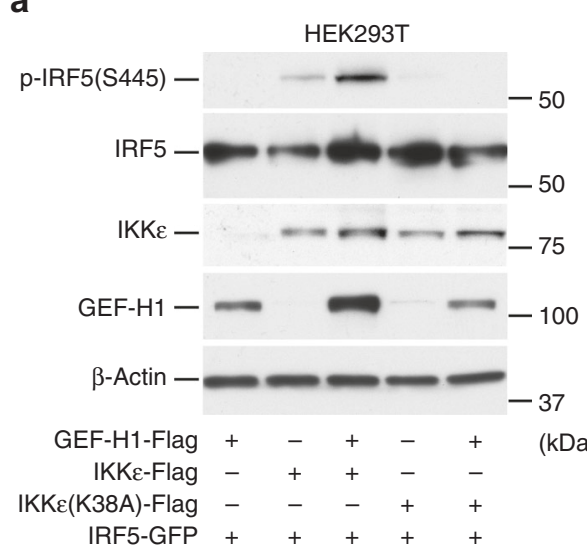

b

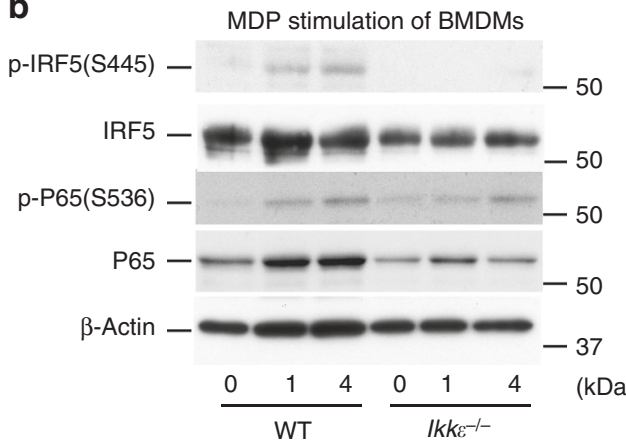

C

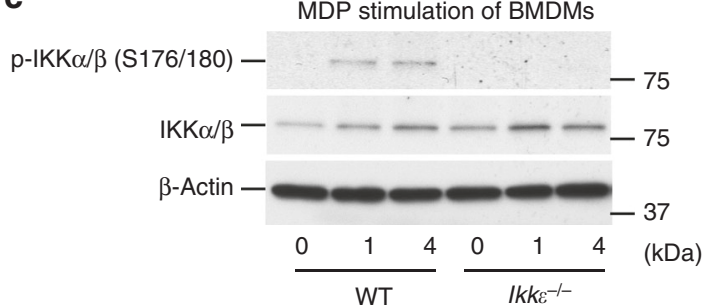

d

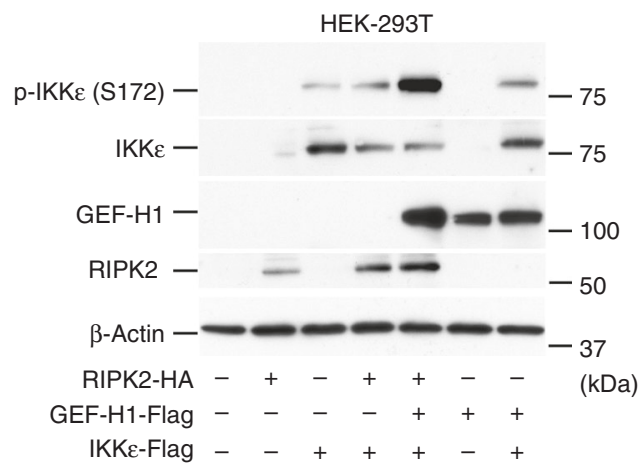

e
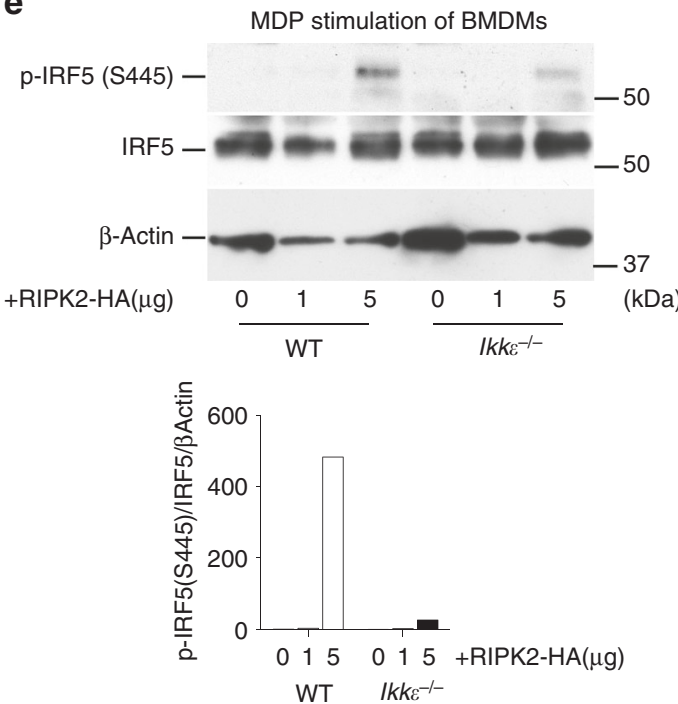

f
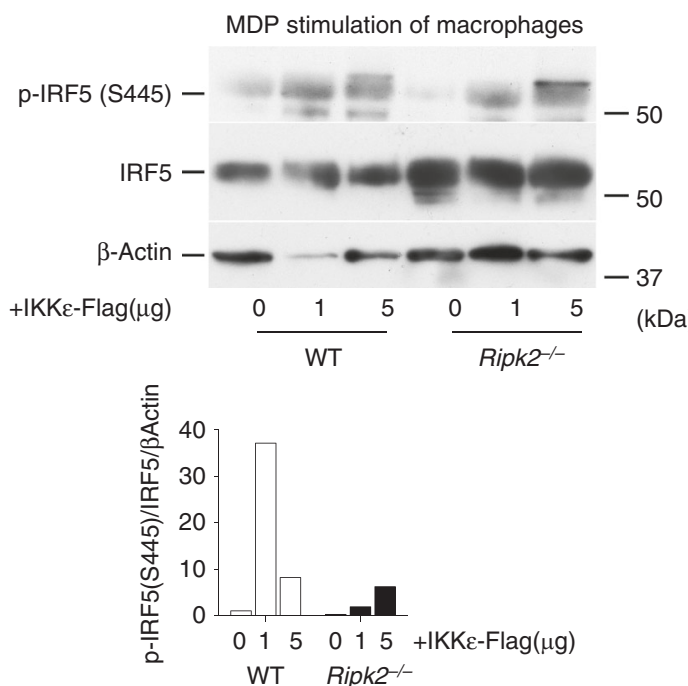

g

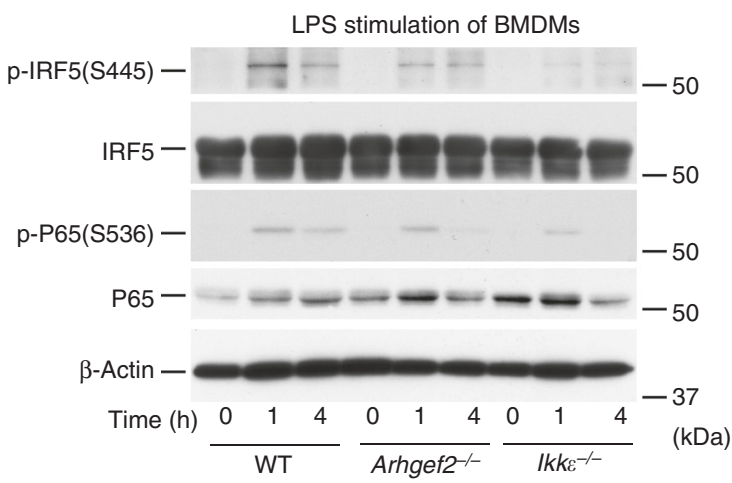

Fig. 2 IKKE is required for the phosphorylation of IRF5. a Assessment of IRF5 phosphorylation in the absence or presence of GEF-H1, IKKE, or a kinase

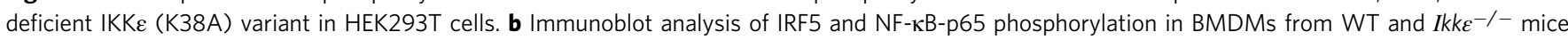
after stimulation with $\mathrm{N}$-glycolyl-MDP for $1 \mathrm{~h}$ and $4 \mathrm{~h}$. c Western blot analysis of IKK $\alpha / \beta$ phosphorylation in BMDMs from WT and $I k k \varepsilon^{-/-}$mice after stimulation with $\mathrm{N}$-glycolyl-MDP for $1 \mathrm{~h}$ and $4 \mathrm{~h}$. d Immunoblot analysis of IKKe phosphorylation mediated by GEF-H1 and/or RIPK2 after co-expression in HEK293T cells. e Immunoblot analysis of IRF5 phosphorylation in response to RIPK2 overexpression in WT and Ikk $\varepsilon^{-/-}$BMDMs with band intensities quantified using densitometry and Image J software. $\mathbf{f}$ Western blot analysis of IRF5 phosphorylation in response to overexpression of IKKE in WT or Ripk2-/- macrophages with band intensities quantified using densitometry and Image J software. $\mathbf{g}$ Assessment of expression and phosphorylation of IRF5 and NF-kB-p65 in BMDMs from WT, Arhgef2-/-, and $I k^{-1} \varepsilon^{-/-}$mice after exposure to LPS $\left(100 \mathrm{ng} \mathrm{ml}^{-1}\right)$ for $1 \mathrm{~h}$ and $4 \mathrm{~h}$. Source data are provided as a Source Data file 
a
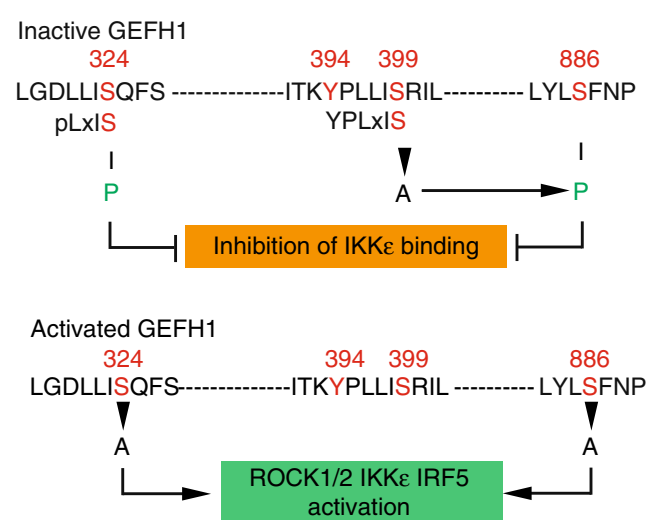

C

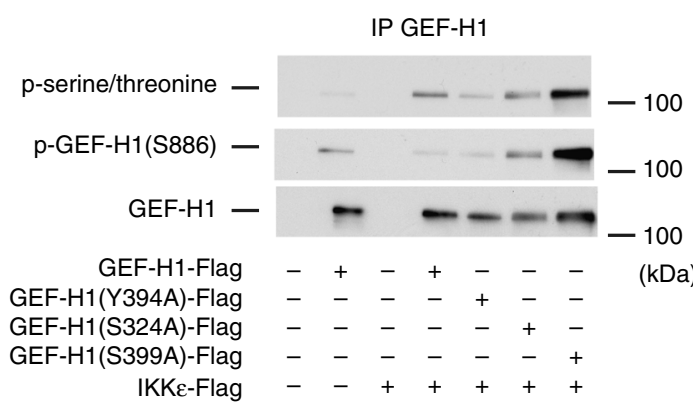

d
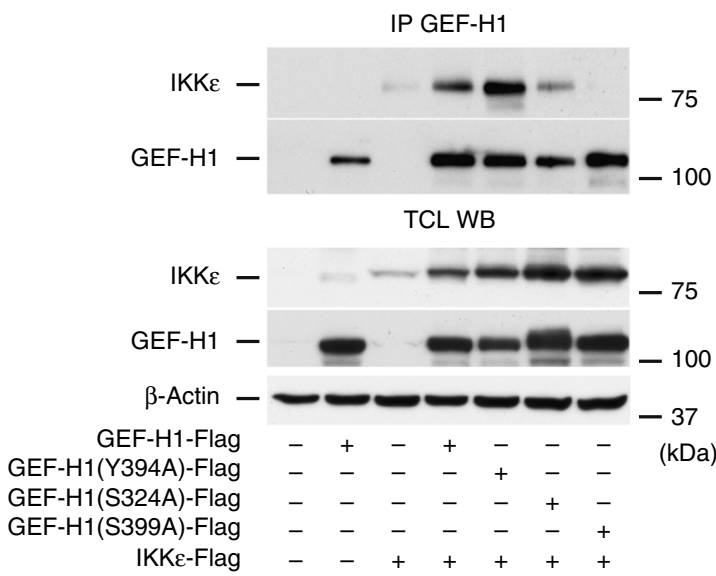

f b

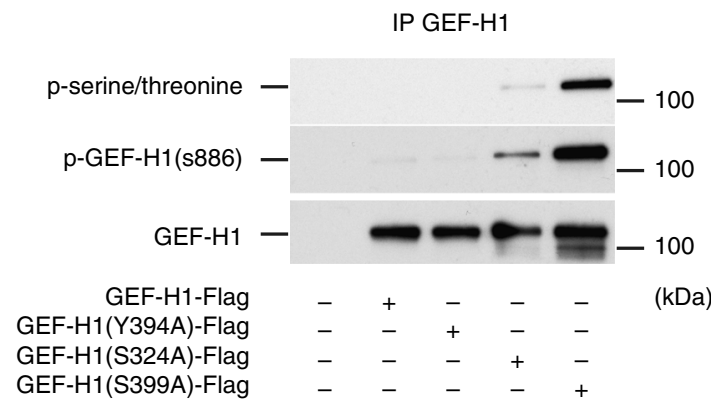

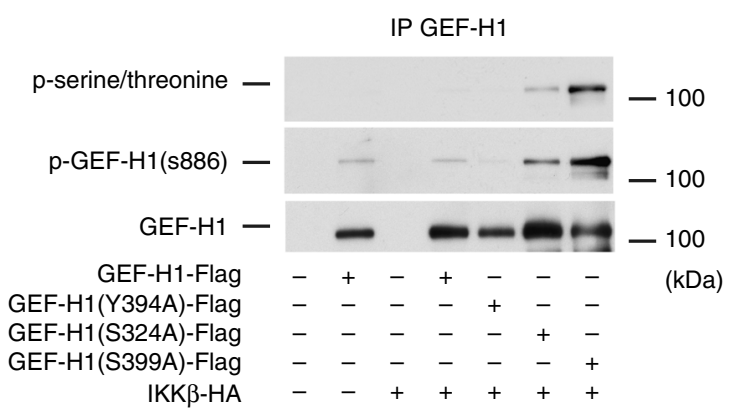

e
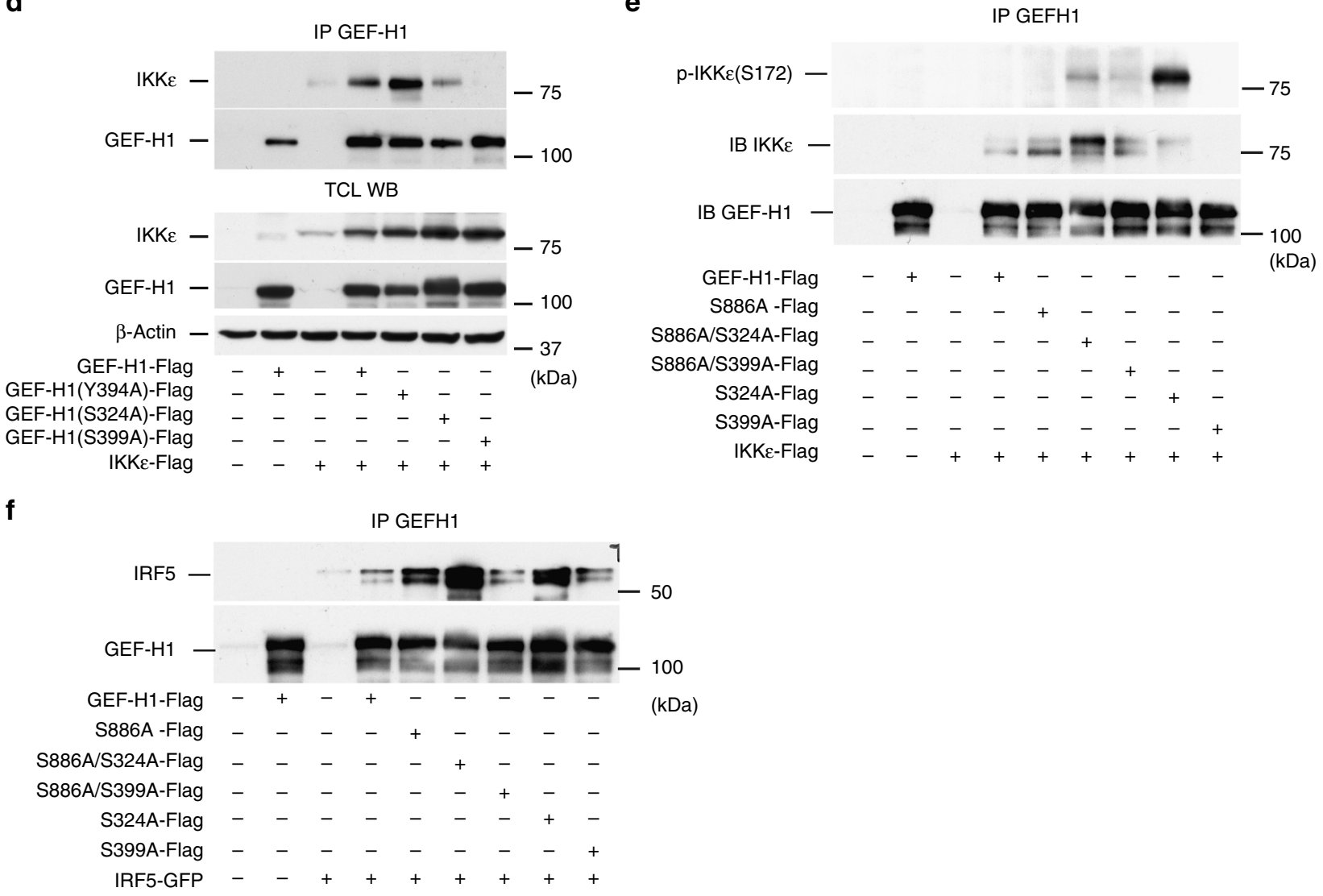

specifically require GEF-H1 and IRF5, we performed highresolution mRNA expression profiling using next-generation RNA sequencing (RNA-seq). We used samples of RNA isolated from BMDMs of Arhgef2 $2^{-1-}$, Irf5 $5^{--}$, and WT mice that were stimulated for $18 \mathrm{~h}$ with MDP. RNA-seq pipeline quantitation in Seqmonk was used on merged transcripts counting reads over exons and $\log$ transformed. Principal component analysis (PCA) of normalized expression revealed that control and MDP-treated WT BMDMs segregated into distinct quartiles, and the control and treated BMDMs lacking Arhgef2 or Irf5 remained clustered close together indicating a reduced transcriptional response (Fig. 5a). The lack of transcriptional changes in response to MDP 
Fig. 3 GEF-H1 dephosphorylation activates IKK $\varepsilon$ and IRF5 binding. a Representation of GEF-H1 amino acid motifs and targeted residues that control dephosphorylation of GEF-H1 for binding and activating IKKe and IRF5. b WT or GEF-H1 variants were expressed in HEK293T cells and the phosphorylation of GEF-H1 assessed after immunoprecipitation with anti-GEF-H1 with pan-phospho S/T ABs or ABs detecting the phosphorylation of GEF-H1 at S886. c Phosphorylation of GEF-H1 and indicated GEF-H1 variants was assessed in HEK293T after immunoprecipitation with anti-GEF-H1 in the presence of either IKKE or IKK $\beta$ with anti-pan-phospho S/T antibodies or ABs detecting the phosphorylation of GEF-H1 at S886. d WT or GEF-H1 variants and IKK $\varepsilon$ were co-expressed in HEK293T cells, GEF-H1 was pulled down with anti-GEF-H1 and co-immunoprecipitated IKKe detected by western blotting. e Amount of phosphorylated and total IKKe that was bound to immunoprecipitated WT and GEF-H1 variants after expression in HEK293T cells. f Co-immunoprecipitation of IRF5 with anti-GEF-H1 antibody in the presence of WT GEF-H1 or GEF-H1 variants expressed in HEK293T cells. Source data are provided as a Source Data file

in Arhgef2 $2^{-1-}$ and Irf5 $-1-$ BMDMs was also revealed in pairwise comparisons in a Cuffdiff analysis where the majority of mRNAs that were upregulated more than twofold by MDP in WT BMDMs failed to transcriptionally activate in Arhgef $2^{-1-}$ and Irf5 ${ }^{-1-}$ BMDMs (Fig. 5b). Hierarchical clustering of MDP regulated genes revealed that GEF-H1 and IRF5 were required for a significant proportion of the transcriptional response induced by MDP (Fig. 5b). Out of the 984 regulated genes with more than twofold regulation upon MDP stimulation in WT BMDMs, GEF$\mathrm{H} 1$, and IRF5 were both required for the induction of 422 or inhibition of 36 transcripts (Fig. 5b, Cluster I and VII, Supplementary Data 1). We observed additional gene clusters that were either dependent on GEF-H1 (cluster IV, 43 genes, Supplementary Data 1) or IRF5 alone (cluster II, 77 genes, Supplementary Data 1). We also detected an additional cluster containing 210 transcripts that were induced by MDP independently of GEF-H1 and IRF5 (Fig. 5b Clusters III and; Supplementary Data 1). For Gene Set Enrichment Analyses (GSEA) genes were further ranked by intensity difference based on their dependence on GEF-H1 or IRF5 and the extent of regulation upon MDP stimulation. Figure $5 \mathrm{c}$ shows the transcripts in cluster I with a $Z$-score $>3.0$ that required both GEF-H1 and IRF5 for induction by MDP. GSEA analysis associated the GEF-H1 and IRF5 dependent gene clustered in the MSigDB Hallmark data base significantly with mesenchymal transition, TNF- $\alpha$ signaling, and Inflammatory responses, and in the MSigDB Canonical pathway (KEGG) database with Focal adhesion, ECM, and cytokine - cytokine receptor interaction (Fig. 5d).

In the next set of experiments, we analyzed the expression of antimicrobial host defense genes induced by MDP in Cluster 1 in macrophages isolated from WT, Arhgef2 $2^{-1-} \operatorname{Irf5} 5^{-1-}$ and $I k k \varepsilon^{-1-}$ mice by qRT-PCR. These experiments confirmed that GEF-H1, IKKe, and IRF5 mediated the expression of peptidoglycan recognition protein 1 (Pglyrp1; Fig. 5e). Pglyrps recognize bacterial cell wall peptidoglycans of Gram-positive and Gramnegative bacteria and have antibacterial functions ${ }^{24-26}$. Additional antimicrobial factor co-regulated by GEF-H1, IKKe, and IRF5 included GranzymeD (GzmD), GranzymeE (GzmE), and Serpine1 all of which have been shown to mediate host defenses ${ }^{27,28}$ (Fig. 5e). Together, these experiments indicated that GEF-H1 and IKKe mediated phosphorylation of IRF5 controlled a unique transcriptional program that could initiate innate immune responses critical for anti-microbial host defense in macrophages through the detection of peptidoglycans and microbial elimination.

The GEF-H1-IKKe-IRF5 signaling axis controls L. monocytogenes. We next determined the role of GEF-H1, IKKe, and IRF5 in the activation of host defense against L. monocytogenes, the causative agent of listeriosis that controls the host-cell cytoskeleton for invasion and intracellular spread ${ }^{29}$. The recognition of $L$. monocytogenes requires peptidoglycan ${ }^{30}$ and nucleic acid sensing receptors ${ }^{31}$. During infection of ARPE-19 cells with GFP expressing L. monocytogenes, RFP-GEFH1 fusion protein redistributed from microtubules into the cytoplasm (Fig. 6a). Exposure to L. monocytogenes induced IRF5 phosphorylation in WT mice but not in Arhgef2-I- or $I k k \varepsilon^{-I-}$ macrophages (Fig. 6b). $I k k \varepsilon^{-1-}$ macrophages were also unable to respond to $L$. monocytogenes with the same level of phosphorylation of NF- $\kappa \mathrm{B}$ p65 that occurred in WT and Arhgef2 ${ }^{-1-}$ macrophages (Fig. 6b). The impaired innate immune activation in macrophages derived

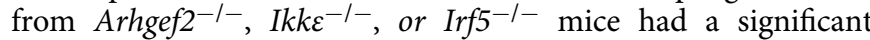
impact on antimicrobial defenses. Macrophages that lacked GEF$\mathrm{H} 1, \mathrm{IKK} \varepsilon$, or IRF5 were significantly more susceptible to infection with L. monocytogenes (Fig. 6c). Exposure to $10^{5}$ bacteria over $2 \mathrm{~h}$ resulted in a 6.9-fold higher bacterial load in Arhgef2 ${ }^{-1-}$, 4.3-fold in $I k k \varepsilon^{-l-}$, and 7.9-fold in Irf5 $5^{-1-}$ macrophages (Fig. 6c). Furthermore, bacterial elimination was particularly lacking in GEFH1-deficient macrophages which were unable to significantly reduce bacterial burden $6 \mathrm{~h}$ after uptake in contrast to WT macrophages. IkkE and Irf5 deficient macrophages were able to reduce intracellular L. monocytogenes by 53 and $74 \%$, respectively. We next determined whether L. monocytogenes induced the transcriptional regulation of genes that were IRF5 and GEF-H1 dependently induced by MDP in macrophages. Indeed, L. monocytogenes induced the expression of mRNA encoding for Pglyrp1, GzmD, and GzmE, and Serpine1 in WT macrophages. The induction of these genes during L. monocytogenes infection was significantly reduced in macrophages that were Arhgef2, Ikke, or Irf5 deficient (Fig. 6d). Together, these data showed that the GEF-H1-IKKe-IRF5 signaling axis was activated as a detection system that defines antimicrobial host defenses in macrophages.

\section{Discussion}

We here demonstrate that GEF-H1 is a critical activator and signaling platform for microbial peptidoglycans recognition that is essential for the phosphorylation of IRF5 by the atypical IKK kinase IKKe. We propose a model in which a GEF-H1 signalosome recruits ROCK1, IKKe, and IRF5, as a prerequisite for IRF5 phosphorylation in response to intracellular peptidoglycan recognition. IKKe is the second atypical IKK kinase that can bind GEF-H1. In the MAVS pathway, GEF-H1 can interact with TBK1 and mediate the phosphorylation of IRF3 and induction of type 1 interferons ${ }^{3}$. However, during the recognition of MDP, GEF-H1 interacted specifically with IKKe for the phosphorylation of IRF5 demonstrating that despite substantial sequence homology the functions of IKKe and TBK1 can be defined by distinct substrate specificities and signaling intermediaries that are immune recognition pathway specific. Previously, it had been difficult to distinguish specific functions of IKKe and TBK1 as they are often simultaneously activated during pathogen-associated molecular pattern recognition ${ }^{32}$.

Our mutational analysis indicates that phosphorylation events at S324, S399, and S886 are critical for the function of GEF-H1. Remarkably, preventing phosphorylation at S399 resulted in the hyper-phosphorylation of GEF-H1 at S886 which prevented the binding to IKKe required for IKKe phosphorylation. Thus, S399 maybe critical for mediating interaction with a phosphatase that 
a

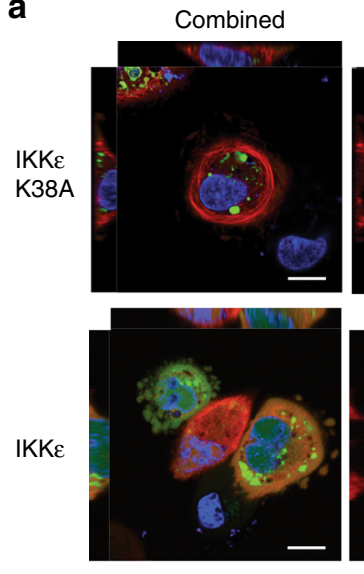

b
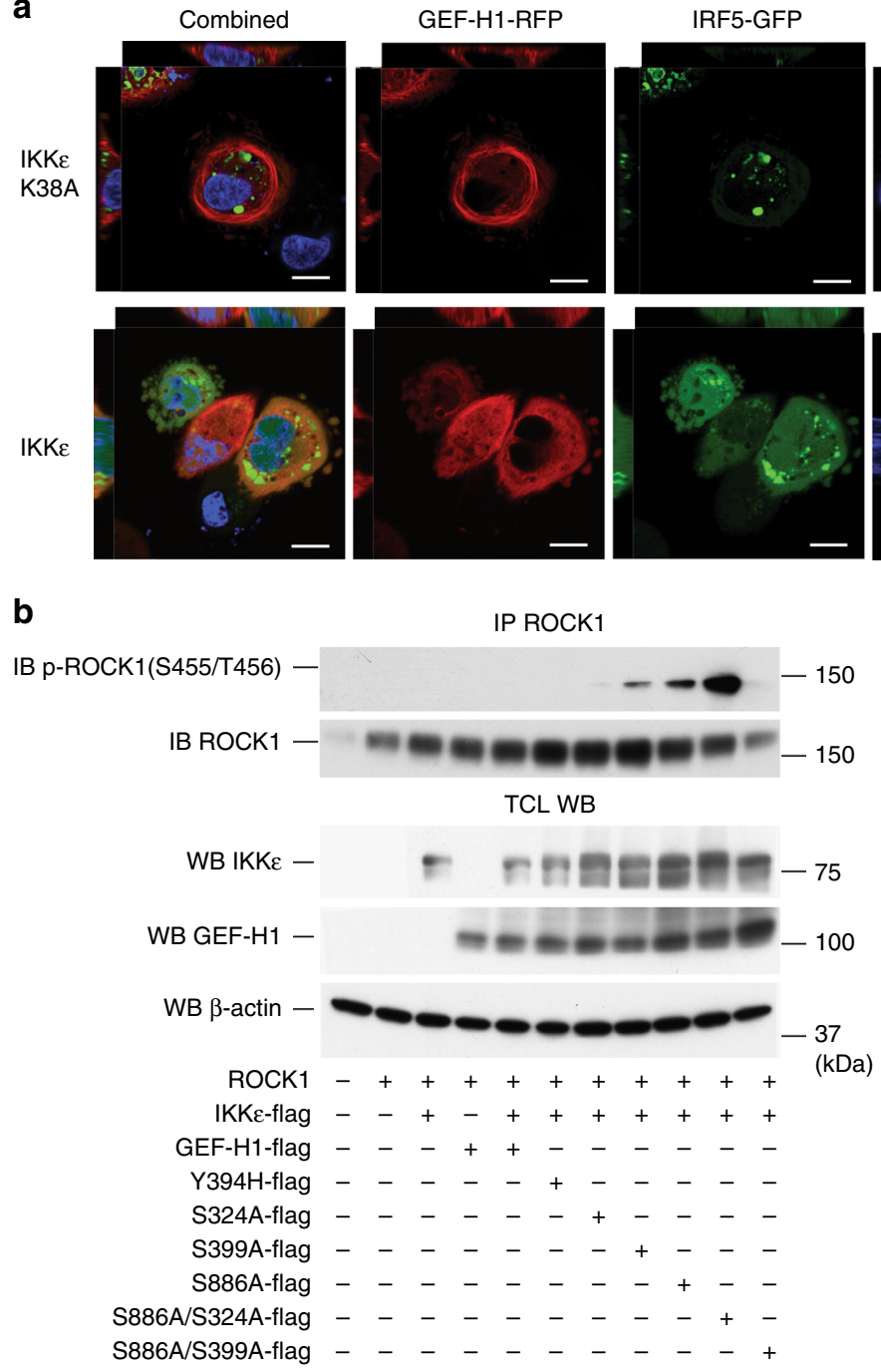

IP ROCK1

d
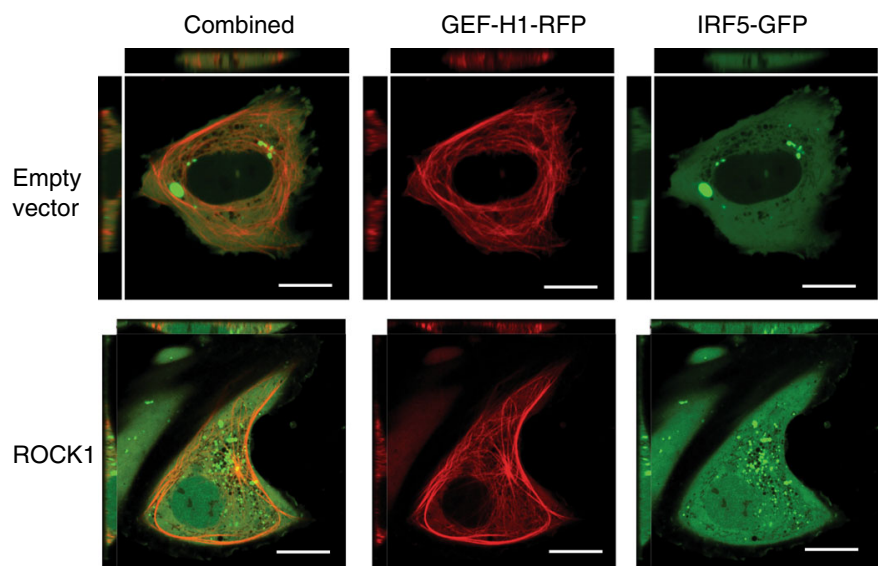
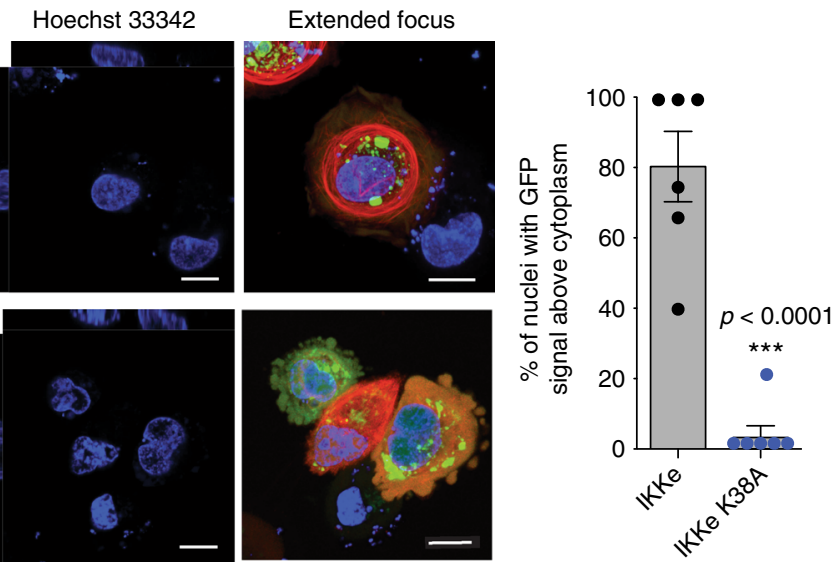

C

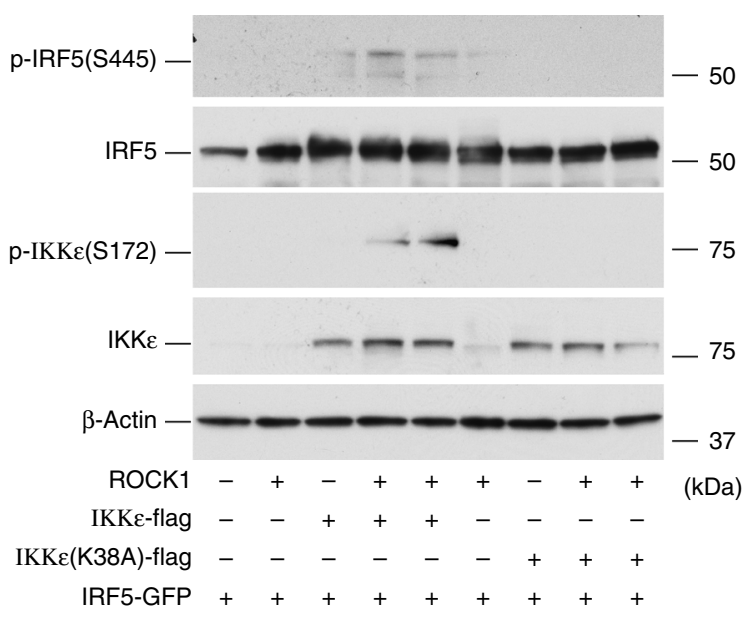

e

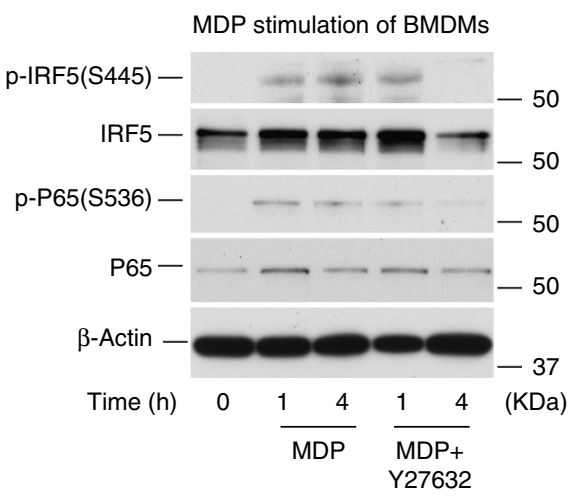

Fig. 4 ROCK1/2 is required for IKKE and IRF5 activation. a Confocal microscopy analysis of primary human ARPE-19 cells $24 \mathrm{~h}$ after transfection with plasmids encoding GFP-tagged IRF5 and RFP-tagged GEF-H1 in presence of IKKE or IKKE (K38A) variant expressing constructs. Nuclear DNA was labeled using Hoechst 33342. Image acquisition was carried out with NIS-Elements imaging software (Nikon) followed by analysis by Volocity (PerkinElmer) to quantify the percentage of nuclei with GFP signal above cytoplasm in either IKKe or IKKe (K38A) transfected cells. Error bars indicate mean \pm SEM. Statistical significance was calculated using Student's $t$-test ${ }^{\star \star \star} P<0.0001(n=6)$. Scale bars, $10 \mu \mathrm{m}$. $\mathbf{b}$ Immunoblot analysis of ROCK1 phosphorylation after immunoprecipitation with anti-ROCK1, in HEK293T cells co-transfected with ROCK1 and IKKE plasmids in presence or absence of WT or GEF-H1 variants. c Western blot analysis of IRF5 and IKK $\varepsilon$ phosphorylation in response to WT or variant (K38A) IKK $\varepsilon$ expression in absence or presence of ROCK1 in HEK293T cells. d Confocal microscopy of ARPE-19 cells that were co-transfected with GFP-tagged IRF5 and RFP-tagged GEF-H1 plasmids in presence or absence of ROCK1 vector. Scale bars, $10 \mu \mathrm{m}$. e Immunoblot analysis of IRF5 and p65 phosphorylation in BMDMs from WT mice after stimulation with $5 \mu \mathrm{g} \mathrm{ml}{ }^{-1}$ of $\mathrm{N}$-glycolyl-MDP with or without $20 \mu \mathrm{M}$ of Y27632 (ROCK1/2 inhibitor). Source data are provided as a Source Data file 


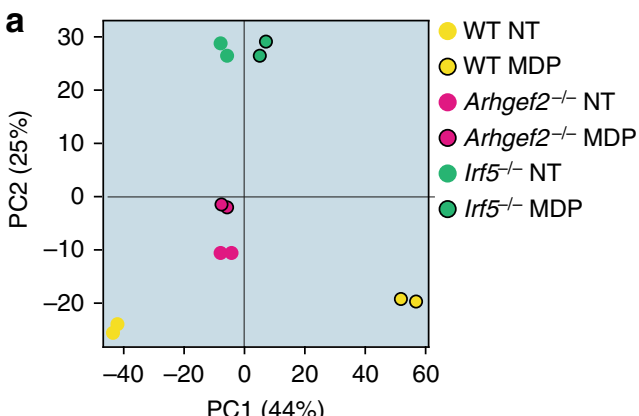

b
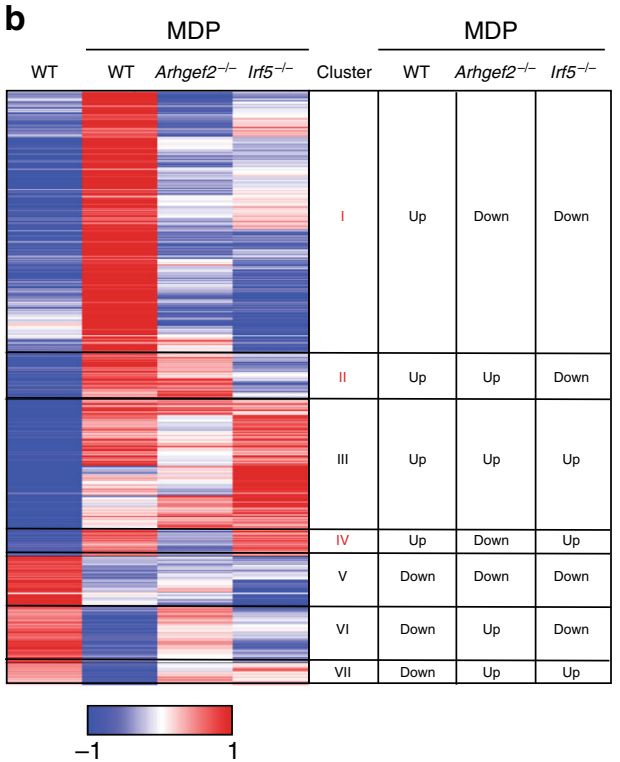

d GSEA/MSigDB-Hallmark

Epithlial mesenchymal transition TNF- $\alpha$ signaling via NFkb Inflammatory response Myogenesis Apical junction E2F targets KRAS siganling Apoptosis Angiogenesis IL2-STAT5 signaling

$$
\begin{array}{lllll}
\hline & 10 & 20 & 30 & 30 \\
-\log (p \text {-value }) &
\end{array}
$$

GSEA/MSigDB-canonical pathway (KEGG)

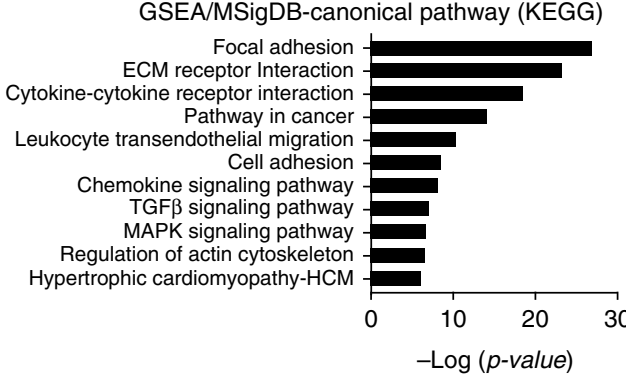

activates GEF-H1 or preventing binding of a kinase that inactivates GEF-H1 by phosphorylation of S886. Phosphorylation of S886 is specifically required for the binding of GEF-H1 to microtubules and dephosphorylation of this residue is required for the release and activation of GEF-H1 $1^{33,34}$. However, we identified S324 as an additional Serine that controls GEF-H1 function. Preventing phosphorylation of S324 and S886 together enhanced GEF-H1 function significantly, dramatically increasing
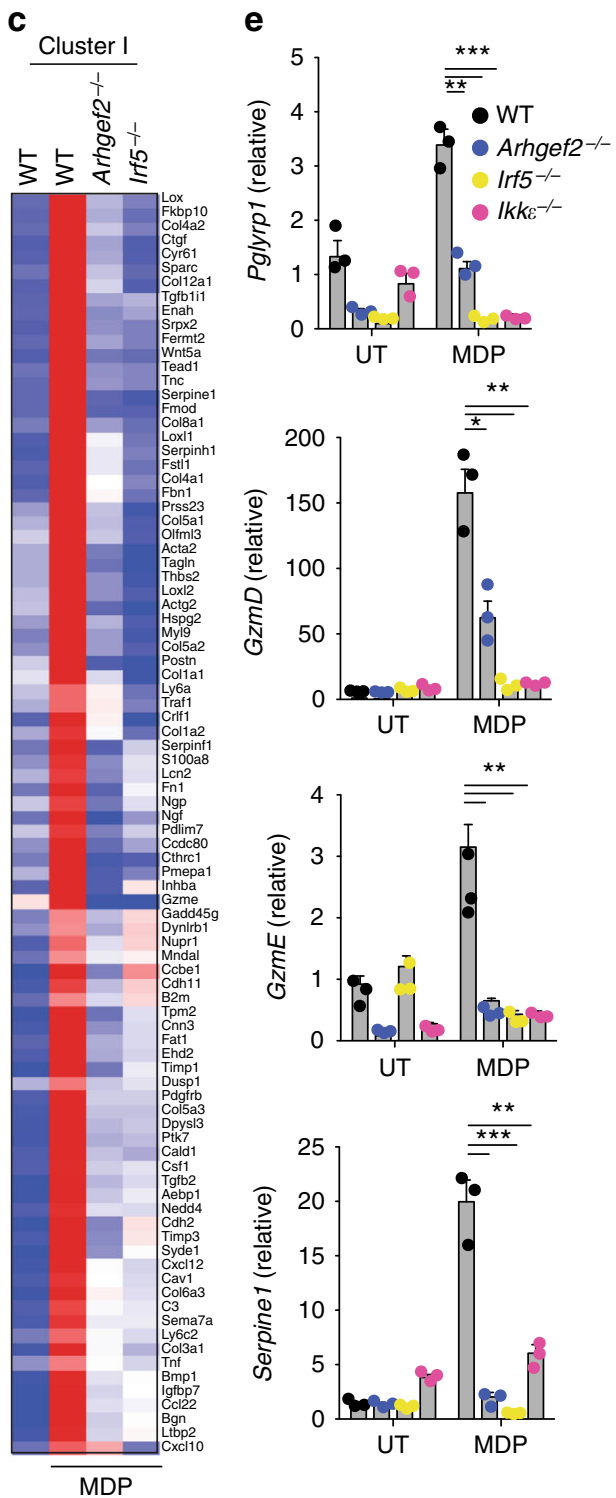
Fig. 5 GEF-H1 and IRF5 control transcriptional programs initiated by MDP. a PCA analysis of variant genes in which input samples are clustered in non-treated (open) and N-glycolyl-MDP stimulated (closed) BMDMs from WT (yellow circles), Arhgef2-/- (pink circles), and Irf5-/- (green circles) mice. b Heat map showing the differential expressed genes (DEG) upon N-glycolyl-MDP stimulation between BMDMs from WT, Arhgef2-/- and Irf5-/- mic. Scale represents Median centered log2FPKM. c Heat map representation of Cluster I shows the N-glycolyl-MDP regulated genes in untreated WT and $N$ glycolyl-MDP treated WT, Arhgef2 ${ }^{-/-}$or Irf5 $5^{-/-}$mice macrophages. Color scales represent d GSEA/MSigDB analysis showing significant pathways in Hallmark and Canonical pathway (KEGG) categories. DEGs were identified using an FDR cutoff $<0.05$ and a fold change cutoff $>2$ by Cuffdiff v1.06 in DNAnexus e, Gene expression analysis by qRT-PCR of Pglyrp1, GzmE, GzmD, and Serpine-1 in BMDMs derived from WT (black square), Arhgef2-/-(blue square), Irf5 ${ }^{-/-}$(yellow square), and $I k_{k \varepsilon^{-/-}}$(pink square), mice after $18 \mathrm{~h}$ stimulation with $\mathrm{N}$-glycolyl-MDP or untreated control (UT). The data are presented as the mean \pm SEM. Statistical significance was tested with Student's $t$-test ${ }^{\star} P<0.05,{ }^{\star \star} P<0.001,{ }^{\star \star \star} P<0.0001(n=3)$. Source data are provided as a Source Data file. RNA sequencing data that support the findings of this study have been deposited in GEO with the accession codes GSE126749. http://www.ncbi.nlm.nih.gov/geo/query/acc.cgi?acc=GSE126749

a

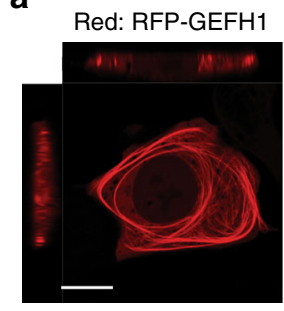

Untreated

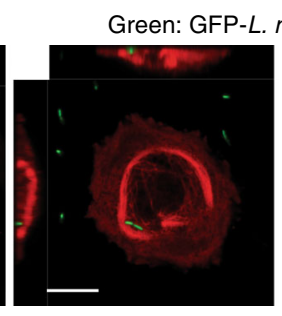

$2 \mathrm{~h}$

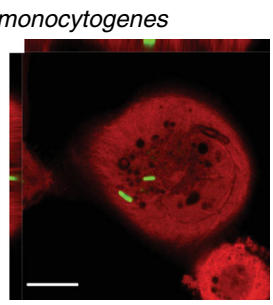

$4 \mathrm{~h}$

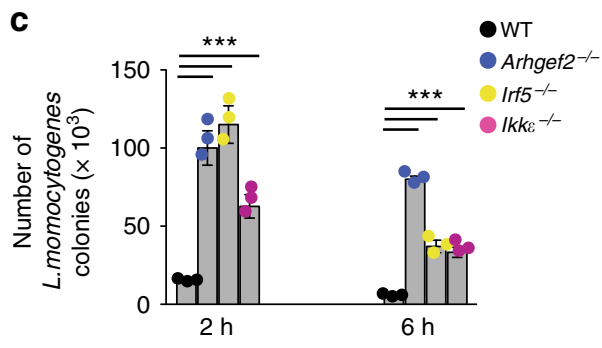

b

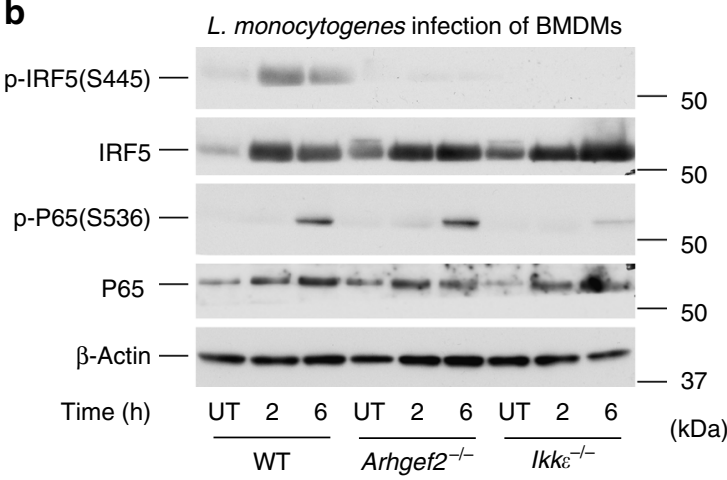

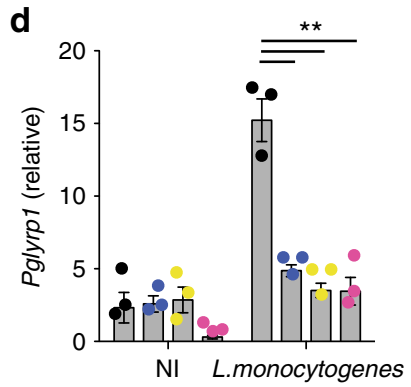
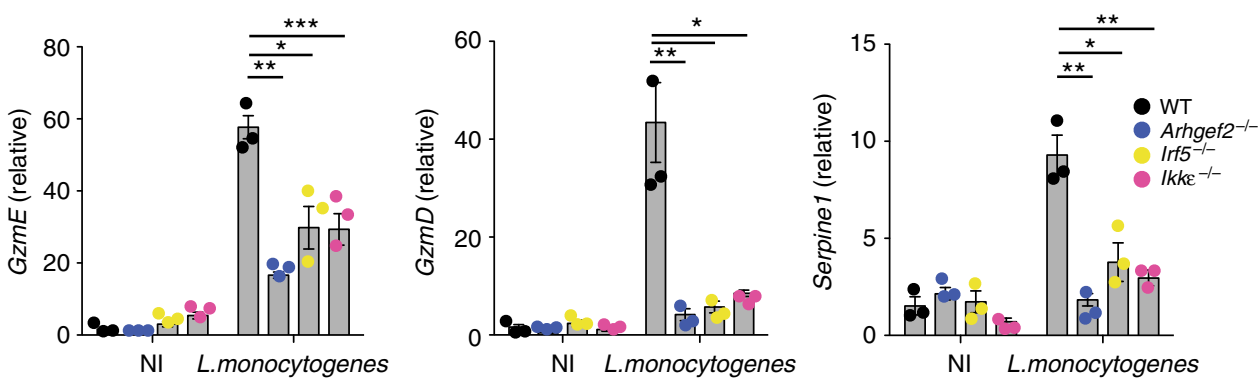

Fig. 6 GEF-H1-IRF5 signaling controls host defense against L. monocytogenes. a Confocal microscopic analysis of subcellular localization of GEF-H1 in ARPE19 before and during infection with L. monocytogenes. Scale bars, $10 \mu \mathrm{m}$. b Immunoblot analysis of N-glycolyl-MDP induced IRF5 and p65 phosphorylation in BMDMs isolated from WT, Arhgef2-/- and $l k k \varepsilon^{-/-}$mice. c Gentamycin protection assay for the assessment of intracellular L. monocytogenes in BMDMs

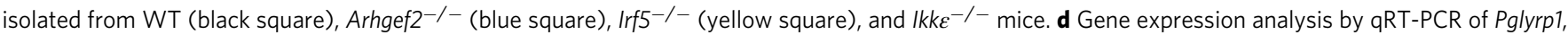

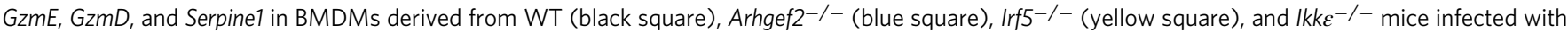
L. monocytogenes or non-infected (NI). Error bars indicate mean \pm SEM. Statistical significance was calculated using Student's two-tailed $t$ test ${ }^{\star \star} P<0.001{ }^{\star \star \star} P<0.0001(n=3)$. Source data are provided as a Source Data file

should be carried out in mutants that prevent inactivation of GEF-H1 through phosphorylation of S886.

We found that ROCK1/2 may be required for IKKe phosphorylation by GEF-H1. The phosphorylation of IKK $\beta$ by RhoA GTPase function for the activation of NF- $\mathrm{BB}$ has been suggested in the TGF- $\beta$ pathway, although in those experiments the responsible GEF had not been identified ${ }^{35}$. It will need to be determined whether ROCK1 or ROCK2 have cell specific function in activating IRF5 as the ROCK inhibitor used in these experiments can inhibit both ROCK kinases. Manipulation of the cytoskeleton by Rho GTPases can activate the NOD-Like receptor signaling pathway ${ }^{36}$ and it needs to be determined whether
GEF-H1 can be activated through $L$. monocytogenes effectors that control the cytoskeleton and impact the microtubule network ${ }^{37}$.

Surprisingly, GEF-H1-mediated IRF5 phosphorylation by MDP occurred in the absence of NOD2, a recognized sensor for $\mathrm{MDP}^{38}$. However, GEF-H1 and NOD2 both are required for the activation of NF- $\kappa \mathrm{B}$, indicating that NOD2 may specifically mediate NF- $\kappa \mathrm{B}-\mathrm{p} 65$ in response to $\mathrm{MDP}^{39}$. Although the role of GEF-H1 in the activation of IKKe was specific for the recognition of MDP, we found that IKKe was also required for TLR4mediated IRF5 phosphorylation that occurs GEF-H1 independent. This indicates that IKKe has a broader unrecognized function in pathways that invoke IRF5-dependent transcriptional 
responses. The specific signaling components that activate IKKe in the TLR pathway for the phosphorylation of IRF5 will need to be identified.

The GEF-H1-IKKe-IRF5 signaling axis was necessary for host defense against the enteroinvasive pathogen $L$. monocytogenes, that leads to a systemic bacterial infection which causes miscarriage in pregnant women, meningitis in neonates as well as the elderly and is often fatal to immunocompromised individuals. Furthermore, Arhgef $2^{-1-}$ macrophages were unable to significantly reduce the bacterial load after uptake, indicating that GEF-H1-mediated defense functions were required for the elimination of intracellular pathogens. Arhgef $2^{-1-}$ macrophages responded with NF-kB-p65 phosphorylation to L. monocytogenes uptake. This may be due to the activation of the STING pathway by microbial nucleic acids during L. monocytogenes infection ${ }^{40}$. Nevertheless, IKKe was of central importance also for the activation of NF- КB-p65 and IRF5 in these experiments. GEF-H1, IKKe, and IRF5 were specifically required for the induction of Pglyrp1 in response to MDP and L. monocytogenes infection. Pglyrps participate in maintaining normal bacterial flora in the gut $^{41,42}$ and are critically involved in regulating inflammatory responses induced by bacteria together with NLRs ${ }^{41}$. However, the precise MDP receptor that activates GEF-H1 induced immune regulation to control innate and cell autonomous responses to commensal and pathogenic microbiota will need to be identified.

Altogether our data indicate that GEF-H1 can promote cell intrinsic innate and cell autonomous immunity by assembling a signalosome that allows the initiation of IRF5-dependent antibacterial transcriptional programs. The GEF-H1-IKKe-IRF5 host defense pathway is essential for the detection of peptidoglycans and enables host defense responses to cope with intracellular pathogens such as L. monocytogenes. In this pathway, GEF-H1 has an essential role for the unique activation of IKKe that has a specific function as an upstream IKKa/ $\beta$ and IRF5 kinase.

\section{Methods}

Cells lines and bone marrow-derived macrophages culture. HEK293T and ARPE-19 cells were purchased from American Type Culture Collection. HEK293T were grown in DMEM and ARPE-19 in DMEM/F12 Medium, supplemented with $10 \%$ fetal bovine serum and $0.5 \%$ penicillin/streptomycin, Bone marrowderived macrophages (BMDMs) cells were generated by flushing bone marrow cells from femurs and tibia of WT or indicated knockout (KO) mice, depleting red blood cells using ACK lysis buffer, and resuspending cell in complete DMEM media supplemented with $10 \% \mathrm{FBS}, 0.5 \%$ penicillin/streptomycin mixture, and $20 \mathrm{ng} \mathrm{ml}^{-1} \mathrm{M}$-CSF. Cells were maintained in culture at $37^{\circ} \mathrm{C}, 5 \% \mathrm{CO}_{2}$ for 6 days before experimentation. BMDMs were stimulated in FBS free DMEM with $N$-Glycolyl-MDP $\left(5 \mu \mathrm{g} \mathrm{ml}^{-1}\right)$ (purchased from Invivogen; Cat\# tlrl-gmdp), LPS-EK (100 $\mathrm{ng} \mathrm{ml}^{-1}$; LPS from E. coli K12; Invivogen) or ROCK1 inhibitor Y27632 $(20 \mu \mathrm{M}$; Abcam) after at least $2 \mathrm{~h}$ of serum starvation. Immortalized WT and Ripk2 deficient macrophages cell lines were cultured in complete DMEM media supplemented with $10 \% \mathrm{FBS}, 0.5 \%$ penicillin/streptomycin.

Mice. Arhgef2 $2^{-1-}$ mice were generated as previously described ${ }^{3}$. C57BL/6 WT (Wild-type), Irf5 $5^{-1-}, \mathrm{Nod}^{-1-}$, and $I k k \varepsilon^{-I_{-}^{-}}$animals were obtained from Jackson Laboratory (Bar harbor, ME). All animals were bred and housed in a pathogen-free animal facility according to institutional guidelines. All experiments were carried out on sex-matched mice at 8-12 weeks old with protocols approved by the subcommittee on Research Animal Care at the Massachusetts General Hospital and Harvard Medical School.

Plasmids. FLAG-tagged GEF-H1 plasmid (Human pCMV6-Entry-GEF-H1) vector was purchased from OriGene. pcDNA3-huIKKe-flag, pcDNA3-huIKKe (K38A)-flag, and pcDNA3.1-huIKK $\beta$-HA plasmids were obtained from Addgene. pCMVtag2c-huNOD2-Flag was gift from Dr. Ramnik J. Xavier. The plasmids pCMV-huROCK1, pcDNA3-huRipK2-HA, and RFP-tagged GEF-H1 were previously described ${ }^{2,3,39}$. pcDNA3-huIRF5-GFP was kindly provided by Dr. Nancy C. Reich Marshall. GEF-H1 and GEF-H1-RFP variants (S324A Y394A, S399A, and S886A), were generated using the Quikchange Site-Directed Mutagenesis Kit (Stratagene) according to the manufacturer's instructions.
Antibodies and western blotting. The following antibodies were used in this study: rabbit antibodies against GEF-H1 (ab155785; 1/2000 dilution), phosphoGEF-H1 (S885(S886), ab94348; 1/2000 dilution), IRF5 (ab21689; 1/1000 dilution) Phospho-ROCK1(T455 /S456, ab203273; 1/1000 dilution), and ROCK1 (EP786Y, ab45171; 1/1000 dilution) were from Abcam. The antibody against phosphoSer445 IRF5 used at 1/2000 dilution was generated by immunizing rabbits with a synthetic peptide (IRLQIpS445NPDLC; NeoBiolab, MA. USA). Phospho-p65NFkB (Ser536, 93H1; 1/1000 dilution), p65-NFkB (D14E12; 1/1000 dilution), phospho-IRF3 (Ser396, 4D4G; 1/1000 dilution), IRF3 (D83B9; 1/1000 dilution), Phospho-IKKa/ß (Ser176/180, 16A6; 1/1000 dilution), IKK 3 (D30C6; 1/1000

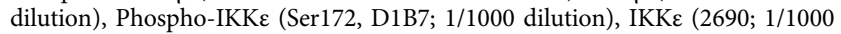
dilution), $\beta$-actin (8H10D10; $1 / 10000$ dilution), and anti-Lamin A/C (4C11; 1/1000 dilution) antibodies were purchased from Cell Signaling Technology. AntiFLAG (F7425; 1/3000 dilution) and anti-HA (H9658; 1/3000 dilution) antibodies were obtained from Sigma. Whole-cell extracts were obtained by harvesting cells with lysis buffer (1\% NP-40, $20 \mathrm{mM}$ Tris- $\mathrm{HCl}$ (pH 7.4), $150 \mathrm{mM} \mathrm{NaCl}, 2 \mathrm{mM}$ EDTA, $2 \mathrm{mM}$ EGTA, $4 \mathrm{mM} \mathrm{Na}_{3} \mathrm{VO}_{4}$, and $40 \mathrm{mM} \mathrm{NaF}$ ) containing protease and phosphatase inhibitors tablets (Roche). Western blotting was performed using standard protocols for SDS-PAGE and wet transfer onto PVDF membranes. Primary antibodies were diluted in blocking buffer (BSA 5\%+1X TBST) and incubated overnight at $4{ }^{\circ} \mathrm{C}$. Secondary anti mouse (NA931V, GE Healthcare) or rabbit (NA934V, GE Healthcare) HRP were used at $1 / 5000$ and incubated for $1 \mathrm{~h}$ at RT. For IP we used true blot anti-rabbit (ROCKLAND; 18-8816-33) or ULTRA anti-mouse (ROCKLAND; 18-8817-33) HRP at 1/4000. The bands were visualized by enhanced chemiluminescence (Western Lightning Plus [PerkinElmer] or SuperSignal West Femto [Thermo Fisher Scientific]) and exposure on film.

Immunoprecipitation and Subcellular fractionation. HEK293T cells were transfected with indicated plasmids using Lipofectamine 3000 (Invitrogen) and Amaxa Mouse Nucleofector ${ }^{\circledast}$ Kit (Lonza, Cat\#VPA-1009) used for Macrophages transfection according to the manufacturer's protocol. The cells were lysed on ice for $20 \mathrm{~min}$, in the same lysis buffer used above to harvest cell lysate for immunoblot. Cell debris was pelleted by centrifugation and the supernatant was then incubated for $30 \mathrm{~min}$ at $4^{\circ} \mathrm{C}$ with protein $\mathrm{G}$ plus agarose (Pierce Thermo Scientific, Rockford, IL) Precleared lysates were incubated at $4{ }^{\circ} \mathrm{C}$ overnight with immunoprecipitation antibodies. The protein $\mathrm{G}$ agarose beads were then added, and the incubation continued for $4 \mathrm{~h}$. Following extensive washes with the same lysis buffer, the agarose beads were mixed with $1 \times$ SDS sample buffer and boiled for $5 \mathrm{~min}$ prior to immunoblotting analysis.

Nuclear and cytoplasmic extracts from BMDMs were prepared using Buffer A (10 mM HEPES, pH7.9; $1.5 \mathrm{mM} \mathrm{MgCl2;} 10 \mathrm{mM} \mathrm{KCl} ; 0.1 \mathrm{mM}$ EDTA; $0.1 \mathrm{mM}$ EGTA; $1 \mathrm{mM}$ DTT; $0.3 \mathrm{mM} \mathrm{Na} 3 \mathrm{VO} 4+$ protease inhibitors tablet [Roche]) and Buffer C (20 mM HEPES, pH7.9; 1.5 mM MgCl2; 1 mM EDTA; 1 mM EGTA; 1 mM DTT; $0.3 \mathrm{mM} \mathrm{Na} 3 \mathrm{VO}$; $0.4 \mathrm{M} \mathrm{NaCl}+$ protease inhibitors tablet [Roche]). In brief, cells were collected by scraping and centrifugation $5 \mathrm{~min}$ at $3000 \mathrm{rpm}, 400 \mu \mathrm{l}$ of Buffer A added to the pellet and after incubation $15 \mathrm{~min}$ on ice, $50 \mu \mathrm{l}$ of $10 \% \mathrm{NP}$ 40 was added and the supernatant collected (cytosolic fraction) after centrifugation at $15,000 \mathrm{rpm}$ for $30 \mathrm{~s}$. A volume $50 \mu \mathrm{l}$ of Buffer $\mathrm{C}$ added to the pellet, vigorously rocked at $4^{\circ} \mathrm{C}$ for $15 \mathrm{~min}$ and centrifuged $5 \mathrm{~min}$ at $15,000 \mathrm{rpm}$. The collected supernatant represents the nuclear extracts. Equal amounts of nuclear protein were loaded in each lane and separated on a $4-20 \%$ Tirs-Gly NuPAGE ${ }^{\circledR}$ gel (Invitrogen), then transferred to a PVDF membrane. Membranes were probed with anti IRF5 antibody or anti-Lamin A/C. Uncropped and unprocessed scans of the most important blots are provided as a Source Data file

Real-time quantitative-PCR. Total RNA from BMDMs was isolated using RNeasy micro kit (Qiagen). cDNA was prepared from RNA using iScript cDNA synthesis kit (Bio-Rad). Real-time PCR was performed using SsoAdvanced ${ }^{\mathrm{TM}}$ Universal SYBR green Supermix (Bio-Rad). The gene expression was normalized to the expression of the gene encoding $18 \mathrm{~S}$. The primer sequences are provided in Supplementary Table 1 .

Bacterial killing assay in vitro. BMDMs derived from WT, Arhgef2 $2^{-1-}$, Irf5 $5^{-1-}$ or $I k k^{-I-}$ mice were plated to 24 -well plate at a density of $1 \times 10^{5}$ cells per well. Cells were infected with GFP-Listeria monocytogenes (generous gift from Dr. John Garber) at a multiplicity of infection (MOI) 1 for $1 \mathrm{~h}$ at $37^{\circ} \mathrm{C}$. BMDMs were washed with sterile HBSS and the extracellular bacteria was eliminated by incubation for $1 \mathrm{~h}$ with $100 \mu \mathrm{g} \mathrm{ml}^{-1}$ gentamicin, Cells were collected (this was considered as time point $2 \mathrm{~h}$ ) or incubated for another $4 \mathrm{~h}$ without gentamicin, which represent the $6 \mathrm{~h}$ time point. After washing with HBSS, BMDMs were disrupted for 15 min with $250 \mu \mathrm{L} \mathrm{dH_{2 }} \mathrm{O}$. Intracellular bacteria were enumerated by serial dilution and spread on Brain Heart Infusion (BHI) agar plates with chloramphenicol.

For western blot assay, BMDMs were seeded at a density of $1 \times 10^{6}$ per well in 12 -well plates. Cells were infected with $L$. monocytogenes at a MOI 1 and harvested at time point $2 \mathrm{~h}$ and $6 \mathrm{~h}$ for protein analysis with immunoblotting.

RNAseq and GSEA analyses. Total RNA was isolated from BMDMs derived from WT, Arhgef2 ${ }^{-I-}$, and Irf5 $5^{-I-}$ mice, using RNeasy Micro kit (Qiagen). Libraries were synthesized using Illumina TruSeq Stranded mRNA sample preparation kit from $500 \mathrm{ng}$ of purified total RNA and indexed adapters according to the 
manufacturer's protocol (Illumina). The final dsDNA libraries were quantified by Qubit fluorometer, Agilent Tapestation 2200, and RT-qPCR using the Kapa Biosystems library quantification kit according to manufacturer's protocols. Pooled libraries were subjected to 35 -bp paired-end sequencing according to the manufacturer's protocol (Illumina NextSeq 500). Targeted sequencing depth was 30 million paired-end reads per sample. Blc2fastq2 Conversion software (Illumina) was used to generate de-multiplexed Fastq files.

Expression values were normalized as Fragments per Kilobase Million reads after correction for gene length (FPKM) in Cuffdiff version 1.06 in the DNAnexus analysis pipleline and filtered for genes that exhibited a statistically significant difference $(P<0.01)$ with a false discovery rate threshold of 0.05 and a biologically relevant change log-fold change $>1$. Samples were analyzed in the RNA-seq pipeline of Seqmonk for mRNAs for opposing strand specific and paired end libraries with merged transcriptome isoforms, correction for DNA contamination and $\log$ transformed resulting expression values in $\log 2$ FPKM. MDP induced mRNAs that were differentially regulated more that twofold (FDR threshold of 0.05 ) in the Cuffdiff analysis of WT BMDMs were imported into Seqmonk for per-probe normalized hierarchical clustering of mRNA transcription in control and $\mathrm{N}$-Glycolyl-MDP stimulated WT and Arhgef2-1- BMDMs.

To generate a ranked gene list for GSEA analyses stranded reads were aligned and counted using STAR (2.5.2a $)^{43}$ in stranded union mode using Illumina's ENSEMBL iGenomes GRCm 38 build and GRCm 38.90 known gene annotations. Count level data were then analyzed using the edgeR Bioconductor package in $\mathrm{R}^{44}$. Filtered genes, expressed at $>1$ count per million (cpm) in at least two samples, were analyzed using the QLF functions comparing untreated and MDP-treated WT and Arhgef2 $2^{-1-}$, Irf5 $5^{-1-}$ BMDMs. All genes were ranked according to their $-\log 10$ transformed corrected $p$-value for differential up/down-regulation by MDP in WT versus Arhgef $2^{-I-}$ and Irf5 $5^{-I-}$ samples. Mouse genes were mapped to their human orthologs using HCOP (http://www.genenames.org/cgi-bin/hcop). The pre-ranked list was used to perform weighted GSEA using the GSEA java application (http:// www.broad.mit.edu/gsea/) that uses the Molecular Signature Database (MSigDB ${ }^{45}$.

Live cell imaging. About 50,000 ARPE-19 cell were plated in Nunc ${ }^{\text {tw }}$ Lab-Tek $^{\text {ma }}$ Chambered Coverglass (Cat.155383PK, Thermo Scientific) and transfected with $200 \mathrm{ng}$ of each indicated plasmid using lipofectamine 3000 .

For GFP tagged-L. monocytogenes experiment, the cells were transfected with RFP-tagged GEF-H1 and infected with a MOI 10. Live cells were imaged with a Nikon A1R-A1 confocal microscope. Image acquisition was carried out with NISElements imaging software (Nikon) followed by analysis with Volocity (PerkinElmer)

Statistical analysis. Error bars indicate mean \pm SEM. All statistical significance was performed with GraphPad Prism Software (version6.01; GraphPad, San Diego, CA) using two-tailed $t$-test. Statistical significance was assumed at $p<0.05$. All experiments were repeated at least two times.

Reporting summary. Further information on experimental design is available in the Nature Research Reporting Summary linked to this article.

\section{Data availability}

RNA sequencing data that support the findings of this study have been deposited in GEO with the accession codes GSE126749. http://www.ncbi.nlm.nih.gov/geo/query/acc.cgi? acc $=$ GSE 126749 .

Received: 17 July 2018 Accepted: 27 February 2019

Published online: 22 March 2019

\section{References}

1. Mostowy, S. \& Shenoy, A. R. The cytoskeleton in cell-autonomous immunity: structural determinants of host defence. Nat. Rev. Immunol. 15, 559-573 (2015).

2. Fukazawa, A. et al. GEF-H1 mediated control of NOD1 dependent NF-kappaB activation by Shigella effectors. PLoS Pathog. 4, e1000228 (2008).

3. Chiang, H. S. et al. GEF-H1 controls microtubule-dependent sensing of nucleic acids for antiviral host defenses. Nat. Immunol. 15, 63-71 (2014).

4. Lazear, H. M. et al. IRF-3, IRF-5, and IRF-7 coordinately regulate the type I IFN response in myeloid dendritic cells downstream of MAVS signaling. PLoS Pathog. 9, e1003118 (2013).

5. Graham, R. R. et al. A common haplotype of interferon regulatory factor 5 (IRF5) regulates splicing and expression and is associated with increased risk of systemic lupus erythematosus. Nat. Genet. 38, 550-555 (2006).

6. Miceli-Richard, C. et al. Association of an IRF5 gene functional polymorphism with Sjogren's syndrome. Arthritis Rheum. 56, 3989-3994 (2007).

7. Krausgruber, T. et al. IRF5 promotes inflammatory macrophage polarization and TH1-TH17 responses. Nat. Immunol. 12, 231-238 (2011).
8. Noppert, S. J., Fitzgerald, K. A. \& Hertzog, P. J. The role of type I interferons in TLR responses. Immunol. Cell Biol. 85, 446-457 (2007).

9. Ren, J., Chen, X. \& Chen, Z. J. IKKbeta is an IRF5 kinase that instigates inflammation. Proc. Natl Acad. Sci. USA 111, 17438-17443 (2014).

10. Mercurio, F. et al. IKK-1 and IKK-2: cytokine-activated IkappaB kinases essential for NF-kappaB activation. Science 278, 860-866 (1997).

11. Zandi, E., Rothwarf, D. M., Delhase, M., Hayakawa, M. \& Karin, M. The IkappaB kinase complex (IKK) contains two kinase subunits, IKKalpha and IKKbeta, necessary for IkappaB phosphorylation and NF-kappaB activation. Cell 91, 243-252 (1997).

12. Rothwarf, D. M., Zandi, E., Natoli, G. \& Karin, M. IKK-gamma is an essential regulatory subunit of the IkappaB kinase complex. Nature 395, 297-300 (1998)

13. Chen, Z. J., Parent, L. \& Maniatis, T. Site-specific phosphorylation of IkappaBalpha by a novel ubiquitination-dependent protein kinase activity. Cell 84, 853-862 (1996).

14. Shimada, T. et al. IKK-i, a novel lipopolysaccharide-inducible kinase that is related to IkappaB kinases. Int. Immunol. 11, 1357-1362 (1999).

15. Peters, R. T., Liao, S. M. \& Maniatis, T. IKKepsilon is part of a novel PMAinducible IkappaB kinase complex. Mol. Cell 5, 513-522 (2000).

16. Pomerantz, J. L. \& Baltimore, D. NF-kappaB activation by a signaling complex containing TRAF2, TANK and TBK1, a novel IKK-related kinase. EMBO J. 18, 6694-6704 (1999).

17. Bonnard, M. et al. Deficiency of T2K leads to apoptotic liver degeneration and impaired NF-kappaB-dependent gene transcription. EMBO J. 19, 4976-4985 (2000).

18. Tojima, Y. et al. NAK is an IkappaB kinase-activating kinase. Nature 404, 778-782 (2000).

19. Pandey, A. K. et al. NOD2, RIP2 and IRF5 play a critical role in the type I interferon response to Mycobacterium tuberculosis. PLoS Pathog. 5, e1000500 (2009).

20. Liu, S. et al. Phosphorylation of innate immune adaptor proteins MAVS, STING, and TRIF induces IRF3 activation. Science 347, aaa2630 (2015).

21. Lopez-Pelaez, M. et al. Protein kinase IKKbeta-catalyzed phosphorylation of IRF5 at Ser462 induces its dimerization and nuclear translocation in myeloid cells. Proc. Natl Acad. Sci. USA 111, 17432-17437 (2014).

22. Zenke, F. T. et al. p21-activated kinase 1 phosphorylates and regulates 14-3-3 binding to GEF-H1, a microtubule-localized Rho exchange factor. J. Biol. Chem. 279, 18392-18400 (2004).

23. Chang, Y. C., Nalbant, P., Birkenfeld, J., Chang, Z. F. \& Bokoch, G. M. GEF-H1 couples nocodazole-induced microtubule disassembly to cell contractility via RhoA. Mol. Biol. Cell 19, 2147-2153 (2008).

24. Royet, J. \& Dziarski, R. Peptidoglycan recognition proteins: pleiotropic sensors and effectors of antimicrobial defences. Nat. Rev. Microbiol. 5, 264-277 (2007).

25. Dziarski, R. \& Gupta, D. The peptidoglycan recognition proteins (PGRPs). Genome Biol. 7, 232 (2006).

26. Royet, J., Gupta, D. \& Dziarski, R. Peptidoglycan recognition proteins: modulators of the microbiome and inflammation. Nat. Rev. Immunol. 11, 837-851 (2011).

27. Harslund, J. et al. The role of Serpine-1 and Tissue inhibitor of metalloproteinase type-1 in early host responses to Staphylococcus aureus intracutaneous infection of mice. Pathog. Dis. 68, 96-104 (2013).

28. Barber, G. N. Host defense, viruses and apoptosis. Cell Death Differ. 8, 113-126 (2001).

29. Cossart, P., Pizarro-Cerda, J. \& Lecuit, M. Invasion of mammalian cells by Listeria monocytogenes: functional mimicry to subvert cellular functions. Trends Cell Biol. 13, 23-31 (2003).

30. Kim, Y. G. et al. The cytosolic sensors Nod1 and Nod2 are critical for bacterial recognition and host defense after exposure to Toll-like receptor ligands. Immunity 28, 246-257 (2008).

31. Abdullah, Z. et al. RIG-I detects infection with live Listeria by sensing secreted bacterial nucleic acids. EMBO J. 31, 4153-4164 (2012).

32. Kumar, H., Kawai, T. \& Akira, S. Pathogen recognition by the innate immune system. Int. Rev. Immunol. 30, 16-34 (2011).

33. Cullis, J. et al. The RhoGEF GEF-H1 is required for oncogenic RAS signaling via KSR-1. Cancer Cell 25, 181-195 (2014).

34. Meiri, D. et al. Modulation of Rho guanine exchange factor Lfc activity by protein kinase A-mediated phosphorylation. Mol. Cell. Biol. 29, 5963-5973 (2009).

35. Kim, H. J., Kim, J. G., Moon, M. Y., Park, S. H. \& Park, J. B. IkappaB kinase gamma/nuclear factor-kappaB-essential modulator (IKKgamma/NEMO) facilitates RhoA GTPase activation, which, in turn, activates Rho-associated KINASE (ROCK) to phosphorylate IKKbeta in response to transforming growth factor (TGF)-beta1. J. Biol. Chem. 289, 1429-1440 (2014).

36. Keestra, A. M. et al. Manipulation of small Rho GTPases is a pathogeninduced process detected by NOD1. Nature 496, 233-237 (2013).

37. Pizarro-Cerda, J., Kuhbacher, A. \& Cossart, P. Entry of Listeria monocytogenes in mammalian epithelial cells: an updated view. Cold Spring Harb Perspect Med 2, (2012). 
38. Ogura, Y. et al. A frameshift mutation in NOD2 associated with susceptibility to Crohn's disease. Nature 411, 603-606 (2001).

39. Zhao, Y. et al. Control of NOD2 and Rip2-dependent innate immune activation by GEF-H1. Inflamm. Bowel Dis. 18, 603-612 (2012).

40. McFarland, A. P. et al. Sensing of bacterial cyclic dinucleotides by the oxidoreductase RECON promotes NF-kappaB activation and shapes a proinflammatory antibacterial state. Immunity 46, 433-445 (2017).

41. Saha, S. et al. Peptidoglycan recognition proteins protect mice from experimental colitis by promoting normal gut flora and preventing induction of interferon-gamma. Cell. Host. Microbe 8, 147-162 (2010).

42. Jing, X. et al. Peptidoglycan recognition protein 3 and Nod 2 synergistically protect mice from dextran sodium sulfate-induced colitis. J. Immunol. 193, 3055-3069 (2014).

43. Dobin, A. et al. STAR: ultrafast universal RNA-seq aligner. Bioinformatics 29, 15-21 (2013).

44. McCarthy, D. J., Chen, Y. \& Smyth, G. K. Differential expression analysis of multifactor RNA-Seq experiments with respect to biological variation. Nucleic Acids Res. 40, 4288-4297 (2012).

45. Subramanian, A. et al. Gene set enrichment analysis: a knowledge-based approach for interpreting genome-wide expression profiles. Proc. Natl Acad. Sci. USA 102, 15545-15550 (2005).

\section{Acknowledgements}

This work was supported by grants DK068181 (H.C.R.), AI113333 (H.C.R.), DK033506 (H.C.R.), and DK043351 (H.C.R.) from the National Institutes of Health.

\section{Author contributions}

H.C.R. designed experiments; and Y.Z., R.Z., S.M.P, N.Y., and P.S. carried out

experiments; Y.Z., R.Z., S.M.P and H.C.R. analyzed and interpreted data, and Y.Z., R.Z, (c) The Author(s) 2019 S.M.P. and H.C.R. wrote the paper.

\section{Additional information}

Supplementary Information accompanies this paper at https://doi.org/10.1038/s41467 019-09283-x.

Competing interests: The authors declare no competing interests.

Reprints and permission information is available online at http://npg.nature.com/ reprintsandpermissions/

Journal peer review information: Nature Communications thanks the anonymous reviewers for their contribution to the peer review of this work. Peer reviewer reports are available.

Publisher's note: Springer Nature remains neutral with regard to jurisdictional claims in published maps and institutional affiliations.

(c) (i) Open Access This article is licensed under a Creative Commons Attribution 4.0 International License, which permits use, sharing, adaptation, distribution and reproduction in any medium or format, as long as you give appropriate credit to the original author(s) and the source, provide a link to the Creative Commons license, and indicate if changes were made. The images or other third party material in this article are included in the article's Creative Commons license, unless indicated otherwise in a credit line to the material. If material is not included in the article's Creative Commons license and your intended use is not permitted by statutory regulation or exceeds the permitted use, you will need to obtain permission directly from the copyright holder. To view a copy of this license, visit http://creativecommons.org/ licenses/by/4.0/. 